\title{
A systematic review of monitoring and evaluation indicators for sexual and reproductive health in humanitarian settings
}

\author{
Elena T. Broaddus-Shea ${ }^{1}$, Loulou Kobeissi ${ }^{2 *}$ (D, Osama Ummer ${ }^{3}$ and Lale Say ${ }^{3}$
}

\begin{abstract}
Objective: To conduct a comprehensive mapping of published indicators for monitoring and evaluation (M\&E) of sexual and reproductive health (SRH) services and outcomes in humanitarian settings.

Methods: A systematic search of the peer-reviewed and grey literature published between January 2008 and May 2018 was conducted to identify all references describing indicator sets for M\&E of SRH services and outcomes in humanitarian settings. The databases MEDLINE, Web of Science, and Global Health, as well as 85 websites of relevant organizations involved in humanitarian response were searched. Characteristics of identified indicator sets and data from individual indicators was extracted.

Findings: Of 3278 records identified, 20 met the review's inclusion criteria and 9 existing indicator sets were identified. A total of 179 relevant indicators were included in the mapping, and removal of duplicates yielded 132 unique indicators. Twenty-seven percent fell within the maternal health domain, followed by the HIV/AIDS domain (26\%) and the gender-based violence domain (23\%). The distribution of indicators by type (process/output, outcome, impact) was balanced overall but varied substantially across domains. The most commonly used data collection platforms were facility-based systems or population-based surveys. Domains covered and indicator definitions were inconsistent across indicator sets.
\end{abstract}

Conclusion: Results demonstrate the need to standardize data collection efforts for M\&E of SRH services and outcomes in humanitarian settings and to critically appraise the extent to which different domains should be covered. A core list of indicators is essential for assessing response status over time as well as across countries.

Keywords: Sexual and reproductive health, Monitoring and evaluation, Humanitarian response, Conflict-affected settings

\section{Background}

In line with target 3.7 of the Sustainable Development Goals (SDGs), access to sexual and reproductive health (SRH) services, including maternal health services, is crucial to ensure health and well-being of all people at all ages, and is a human right [1]. Yet ensuring access to SRH services is particularly challenging in humanitarian settings, given the collapse of health systems, limited

\footnotetext{
* Correspondence: kobeissil@who.int

${ }^{2}$ Department of Reproductive Health and Research, World Health

Organization, Geneva, Switzerland

Full list of author information is available at the end of the article
}

quality of care and availability of human resources, as well as the increased vulnerabilities associated with conflict and displacement.

According to the Inter-agency Field Manual for Reproductive Health in Crisis, a humanitarian setting is "... one in which an event or series of events has resulted in a critical threat to the health, safety, security or well-being of a community or other large group of people. The coping capacity of the affected community is overwhelmed and external assistance is required. This can be the result of events such as

C The Author(s). 2019 Open Access This article is distributed under the terms of the Creative Commons Attribution 4.0 International License (http://creativecommons.org/licenses/by/4.0/), which permits unrestricted use, distribution, and 
armed conflicts, natural disasters, epidemics or famine, and often involves population displacement [2]".

The Inter-Agency Working Group (IAWG) for reproductive health in crises provides guidance on six main objectives around the minimum initial service package (MISP) for reproductive health in crisis [2]. The MISP is a set of priority activities intended to be implemented immediately at the onset of crisis. The MISP also forms part of the Sphere Project's minimum standards for humanitarian assistance [3]. Despite these established international standards for basic service provision in humanitarian settings, there remains no consensus around monitoring and evaluation (M\&E) frameworks or sets of indicators to assess adequacy of SRH service provision in humanitarian settings as well their respective impacts on associated morbidity and mortality. Moreover, as time passes after the initial onset of an emergency and the setting passes into extended (or protracted) stages of crisis, service provision should move towards more comprehensive coverage of SRH needs [2]. Although M\&E indicators and standards play an important role in guiding the transition to more comprehensive service provision, there are currently no widespread standards regarding core indicators that should be collected in extended stages in emergency settings versus those for acute stages.

Valid, timely, and reliable monitoring and evaluation data is essential for guiding effective humanitarian response as well as ensuring the accountability of all actors involved. Yet, often even the minimal needed data is unavailable [4]. Improving data availability and quality in humanitarian settings will require the commitment and willingness of the humanitarian actors across diverse agencies and organizations to invest in the time, effort and platforms to allow for the needed data to be collected. It will also require an openness for greater consistency in data collection, analysis, and use [4], in order to ensure comparability across settings and to demonstrate performance expectations for implementing organizations [5].

Given the need for increased focus on and consistency in the M\&E of SRH services in humanitarian settings, the World Health Organization's (WHO) Department of Reproductive Health and Research, in collaboration with the Department of Maternal, Child and Adolescent Health as well as numerous partner organizations and agencies, has committed to guide a collaborative and consultative review process. Ultimately, the goal is to propose a standardized set of core indicators for M\&E of SRH services and outcomes in acute and extended humanitarian settings, and to provide guidance on their use. Initiated in April 2018 and expected to conclude in 2020, the review process consists of identifying current M\&E indicators and mechanisms for SRH in humanitarian settings and convening in-depth stakeholder consultations to: assess their adequacy; standardize definitions and data collection procedures; and select and prioritize indicators for inclusion in a set of recommended indicators.

The process began with a systematic literature review conducted to identify current M\&E indicators. An initial technical consultation which convened a wide variety of experts and other stakeholders was then held in December of 2018. The final step in the review process will involve field testing of standardized indicators and accompanying implementation recommendations in a variety of settings impacted by differing types and stages of humanitarian crises (April 2019-June 2020). Field testing will assess feasibility and allow for finalization of the core indicator sets across the different SRH domains, including establishing subsets specific to acute and extended stages of emergency.

\section{Main text}

This paper seeks to describe the systematic literature review, which began this multi-year process and is intended to improve quality and consistency in the M\&E of SRH services in humanitarian settings. This literature review served as the first step in the broader process and was conducted to describe and assess existing indicators published in the peer-reviewed and grey literature for SRH services and outcomes in humanitarian settings. Thus, it aimed to achieve the following objectives:

1. Identify existing indicator sets described within the peer-reviewed and grey literature, which are intended for the monitoring and evaluation of SRH services and outcomes in humanitarian settings.

2. Examine all relevant individual indicators within each set in order to assess the relative coverage of different SRH domains and topics, the relative frequency of indicator types (i.e. process, output, outcome, or impact), and to identify commonly occurring indicators.

\section{Methods}

This review was conducted in accordance with the preferred reporting items for systematic review and metaanalysis protocols (PRISMA-P) guidance [6].

\section{Eligibility criteria}

References published in the peer-reviewed literature, the grey-literature, and on websites were eligible for inclusion if they

- Described indicators for monitoring and/or evaluation of SRH in humanitarian settings

- Addressed multi-domain SRH services and outcomes 
- Were published in English

- Were published after January 1st, 2008

\section{References were excluded if any of the following criteria were relevant}

- Not specific to humanitarian settings

- Not specific to SRH

- Addressed only a single SRH domain

- Did not describe specific indicators for monitoring or evaluating SRH services

- Described research other than monitoring and/or evaluation (i.e. needs assessments, retrospective analyses of DHS data)

- Described monitoring and/or evaluation of SRHrelated interventions and services that were not health system-based (i.e. cash transfer program evaluations)

Humanitarian settings were defined according to the definition noted above from the Inter-agency Field Manual for Reproductive Health in Crisis. For the purposes of this review, SRH domains were defined in line with the MISP objectives from the Inter-Agency Field Manual on Reproductive Health in Humanitarian Settings, i.e.: Adolescent Reproductive Health (ARH), Family Planning (FP), Maternal Health (MH), Comprehensive Abortion Care (CAC), Gender-based Violence (GBV), Sexually Transmitted Infection (STI), and HIV/AIDS (HIV) [2]. The reason for including only references that addressed multiple (two or more) domains was due to the fact that even at the most minimal (such as the service package described in the MISP) SRH service provision in humanitarian settings must cover multiple domains. This inclusion criteria ensured that indicator sets identified in the review were those intended for assessing multi-domain SRH service packages, as opposed to siloed programs focused on a single domain. Date criteria were applied to ensure that materials retrieved reflected up-to-date practices and perspectives on monitoring and evaluation as well as of SRH.

\section{Information sources}

Databases searched for peer-reviewed literature included: MEDLINE/PubMed, Web of Science, and Global Health. To identify grey-literature and online resources, a manual search was conducted of the websites of organizations that work extensively in humanitarian settings and/or do extensive work in the area of SRH.

\section{Search strategy}

For the database search, search terms were selected by identifying relevant medical subject headings (MeSH) and keyword terms for the following concepts: sexual, reproductive, and maternal health; humanitarian settings; and M\&E. The initial search was constructed in PubMed using "OR" to link terms for the same concept, and the term "AND" to link the groups of terms for different concepts. This was then translated into the correct syntax for the other two databases. Filters were applied to all searches to retrieve articles published in English since January 1st, 2008. The full search syntax for each database is available in the Additional file 1.

For the online search, an initial list of 60 organizations was compiled based on a list of participating agencies within the WHO Global Health Cluster. As potentially relevant web content and documents were identified while searching the websites of these organizations, the names of additional organizations mentioned (for example, collaborating partners on an initiative, or co-authors on a document) were recorded. The websites of these additional organizations were then searched as well. In total, 85 websites were searched (see Additional file 1 for complete list).

\section{Data management and selection process}

Title, abstracts and other reference information for hits identified via the database search were downloaded to EndNote, and then exported in spreadsheet format. During the online search, all potentially relevant references were either downloaded as PDFs or saved as screenshots, and the bibliographic information for each (title, date, author, etc.) was entered into a spreadsheet. Two reviewers then independently screened the titles and abstracts of all peer-reviewed references and screened online references. Discrepancies in decisions about whether to include or exclude a particular reference were resolved through discussion. Next, the full-text of all references included during the initial round of screening were retrieved and reviewed. During this round of screening, reasons for exclusion were recorded and the list of references to include in the review was finalized.

\section{Data extraction \& synthesis}

First, metadata for indicator sets described was extracted from all references selected for inclusion during screening. This included: citation and name of indicator set, intended setting and stage of emergency, SRH domains examined, data sources used for indicators, and supporting resources available. Data for individual indicators were then extracted only for indicators that met the following criteria: 1) were specific to the health sector, 2) fell into one of the six SRH domains addressed by MISP objectives, and 3) could be defined in terms of specific, objective, and comparable numerators and denominators. These criteria were applied because the goal of this review was to identify indicators that would be comparable over time, across settings, and 
across emergency types. Finally, detailed information was extracted for each relevant indicator within the indicator sets identified. This included: source, domain, topic, name of indicator, definition, data source, and data collection method. Additionally, indicators were compared to those included in the monitoring and evaluation frameworks for the SDGs, the Global Strategy (GS) for Women's Children's and Adolescents' Health, and WHO's 100 Core Health Indicators [7-9]. Finally, indicators were classified by type (process/output, outcome, and impact), in line with the WHO Health Emergencies Program (work stream 4 on standardized indicator sets for acute and protracted event monitoring).

\section{Results}

\section{Search results}

As shown in Fig. 1, 3,470 records were retrieved from the database search, which resulted in 3155 unique hits after duplicates were removed. An additional 123 potentially relevant records were identified through online searching, yielding a total of $\mathbf{3 2 7 8}$ records for screening. Of these, 3237 were excluded during the initial round of screening, and another 21 were excluded during full text screening. In total, 20 references were included in the analysis [3, 10-28]. From these 20 references, 9 existing indicator sets were identified. Finally, 179 relevant indicators from the indicator sets identified were included in the mapping. Removal of duplicates yielded 132 unique indicators.

Table 1 describes the 9 indicators sets that were identified from references included in the review (details for each reference are available in the Additional file 1). Table 2 provides the full list of unique indicators identified, organized by domain.

\section{Coverage of SRH domains and topics within domains}

As shown in Table 1, all indicator sets included indicators on $\mathrm{MH}$, and all but one included indicators on GBV. Domains with the least coverage were those reporting on ARH and CAC. When looking at individual indicators, a similar trend emerged. The majority (27\%) of the 132 unique indicators identified fell within the MH domain, followed by the HIV domain (26\%) and the GBV domain (23\%). Domains with the least coverage were ARH (3\%) and CAC (3\%).

For all domains other than ARH and CAC, indicators were also broken down by topic. Distributions by topic are shown in Fig. 2. Topics with the greatest coverage overall were prevention of mother-to-child transmission (PMTCT) $(n=16)$ within the HIV domain, and occurrence of violence $(n=11)$ within the GBV domain. Within other domains topics with the most coverage were STI service availability and STI incidence and prevalence (both $n=4)$, MH emergency care $(n=5)$, and use of contraception $(n=6)$.

The domain with the greatest breadth (number of different topics) was $\mathrm{MH}$ which had indicators covering 11 different topics. The number of indicators per topic was low, however, with topics covered by between 2 and 5 indicators. In contrast, the domains of GBV and HIV each included fewer topics (8 and 7, respectively) but had more indicators clustered within specific topics (occurrence of violence and PMTCT). The STI and FP domains had the fewest topics-3 and 4 respectively.

\section{Indicator types}

Overall the distribution of indicators by type (i.e. process/ output, outcome, or impact) was fairly balanced, with the majority classified as outcome (41\%), followed by impact (30\%), and then by process/output (30\%). When disaggregated by domain, as shown in Fig. 3, distributions of indicators by type varied substantially across domains. The greatest number of Impact indicators were in the GBV domain $(n=16)$, followed by the MH domain $(n=12)$. Numbers of outcome indicators were greatest in the HIV domain $(n=20)$ and in the MH domain $(n=15)$. These two domains also included the greatest number of process/ output indicators ( $n=11$ and $n=9$, respectively).

\section{Intended context for use}

Of the 9 indicator sets identified, 6 were intended for use in all humanitarian settings, 1 was designed specifically for conflict-affected settings, 1 was designed for post-disaster settings in the United States, and 1 was intended for use with displaced populations in both camp and urban settings, with separate versions available for the two settings. Regarding stage of emergency, 6 indicator sets were intended for use during both acute and extended stages, 2 were intended specifically for the acute stage, and 1 was intended for extended or protracted stages. Interestingly, of the 6 indicator sets that indicated that they were appropriate for both acute and extended emergency stages, none specified which of the indicators included were appropriate during which stages.

\section{Data sources used}

The majority of indicators $(n=65)$ used data only from facilities, meaning data obtained directly from facility records, entered into reporting systems by facility staff, or collected during facility assessments. Fifty indicators used data only from the affected population, obtained via population-based surveys. Five indicators could be calculated using data from either facilities or affected populations, depending on which definition was used for the indicator. For example, 'complete antenatal care (ANC) coverage' could be 

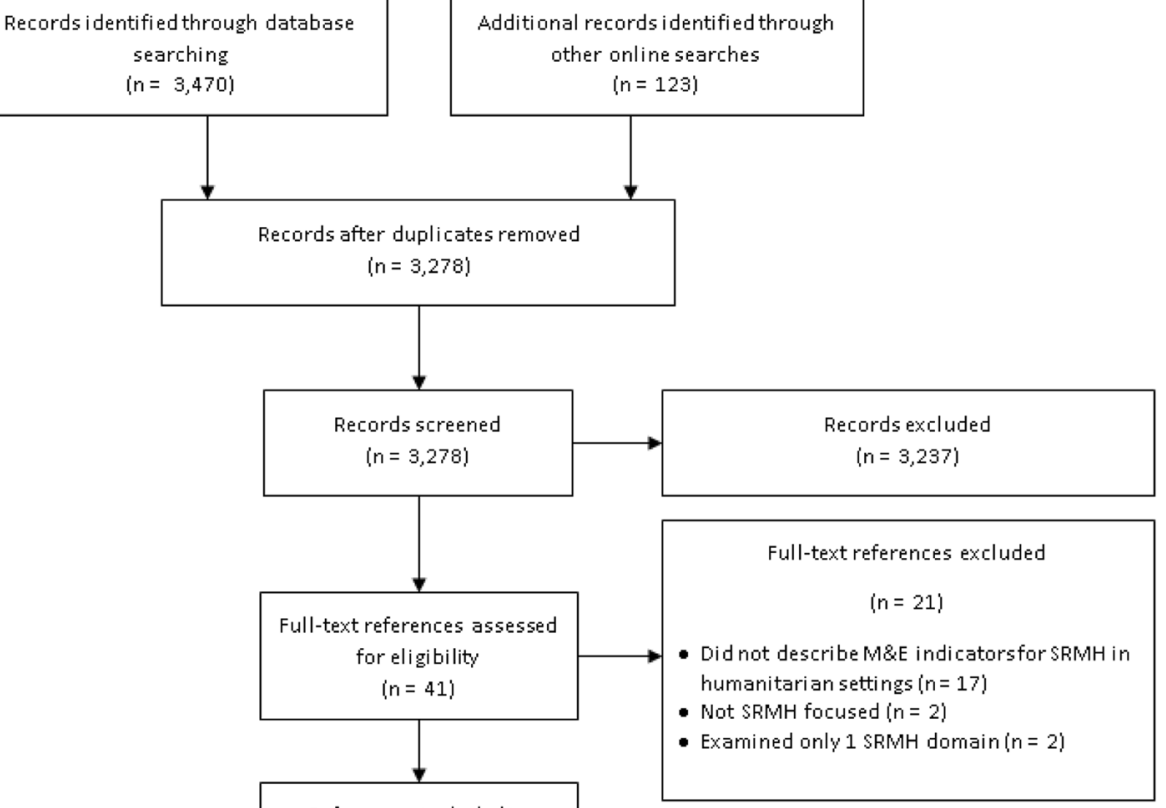

References includedin

analysis

$(n=20)$

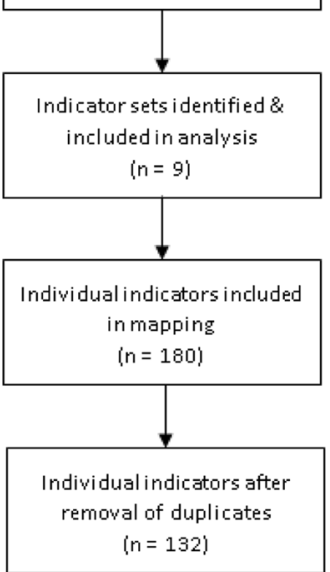

Fig. 1 PRISMA Flowchart showing resource selection, indicator set identification, and indicator mapping process. ${ }^{1} \mathrm{ARH}$ and $\mathrm{CAC}$ indicators not included since coverage of these domains is limited

obtained using facility data when defined as, "percentage of total number of live births in which the mother made at least four ANC visits during the antenatal period at the time of delivery at facility," but would require data from a population-based survey to calculate when defined as, "percentage of all women whose most recent pregnancy ended in a live birth or stillbirth in the last two years who received at least three ANC care visits by a trained provider."

Aside from the indicators drawing on facility or population data, three indicators used data from program records. This includes, for example, the indicator on clean delivery kit coverage-this is intended to be calculated using data from the program distributing the kits on the total number distributed. Two indicators used data obtained directly from service providers regarding their knowledge and training. For seven indicators, it was unclear what data source should be used, and the set they were included in did not specify.

\section{Frequently occurring indicators \& overlap with priority indicators}

As shown in Tables 2 and 3, a total of 33 indicators appear in multiple sets. Of these, however, only 20 have definitions which are consistent across sets. As shown in Tables 2 and 4, 28 indicators overlapped with those included in the monitoring frameworks for the SDGs or the Global Strategy (GS), or in the WHO's 100 Core Health Indicators (Core WHO). Less than half (only 11) 


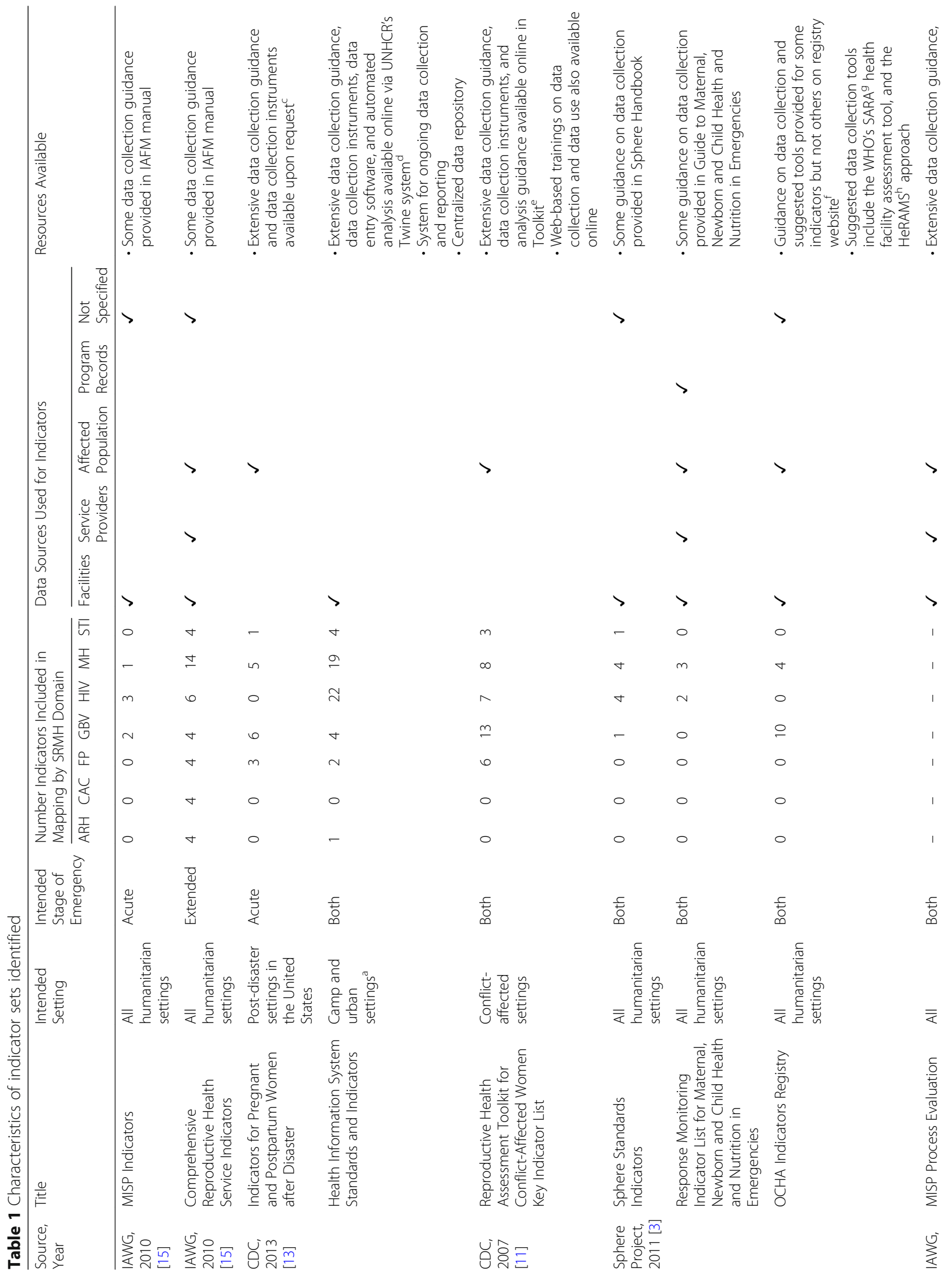




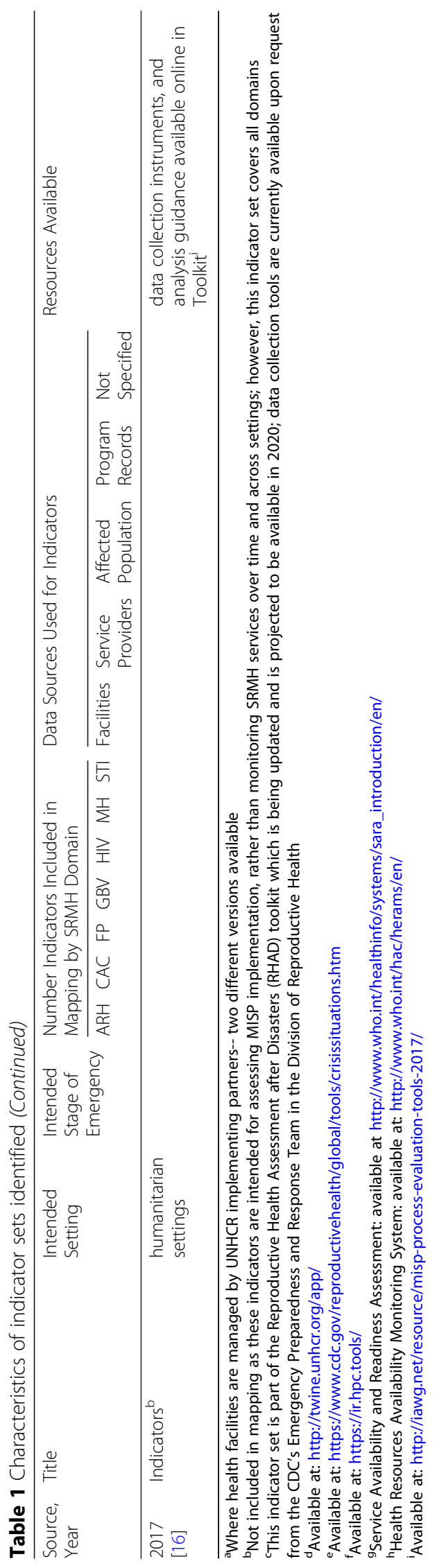


Table 2 Unique indicators identified $(n=132)$

\begin{tabular}{|c|c|c|c|c|c|c|c|c|c|c|c|}
\hline \multirow[t]{2}{*}{ Domain } & \multirow{2}{*}{$\begin{array}{l}\text { Indicator (Sets it Appears } \\
\text { In) } \\
\text { [italics = appears in } \\
\text { multiple sets }]\end{array}$} & \multirow[t]{2}{*}{ Definition (Sets) } & \multirow[t]{2}{*}{ Type } & \multicolumn{5}{|c|}{ Data Source } & \multicolumn{3}{|c|}{$\begin{array}{l}\text { Overlap with } \\
\text { Priority } \\
\text { Indicators }\end{array}$} \\
\hline & & & & Facilities & $\begin{array}{l}\text { Service } \\
\text { Providers }\end{array}$ & $\begin{array}{l}\text { Affected } \\
\text { Population }\end{array}$ & $\begin{array}{l}\text { Program } \\
\text { Records }\end{array}$ & $\begin{array}{l}\text { Not } \\
\text { Specified }\end{array}$ & SDG & GS & $\begin{array}{l}\text { Core } \\
\text { WHO }\end{array}$ \\
\hline ARH & $\begin{array}{l}\text { Condom use among } \\
\text { young people (b) }\end{array}$ & $\begin{array}{l}\text { Percentage of sexually } \\
\text { active young people who } \\
\text { reported using a condom at } \\
\text { last intercourse }\end{array}$ & Outcome & & & $\checkmark$ & & & & & \\
\hline ARH & $\begin{array}{l}\text { Incidence of STDs in } \\
\text { young people (b) }\end{array}$ & $\begin{array}{l}\text { Number of reported cases } \\
\text { of STDs among young } \\
\text { people by the specified } \\
\text { time period per } 1000 \text { young } \\
\text { people }\end{array}$ & Impact & $\checkmark$ & & & & & & & \\
\hline ARH & $\begin{array}{l}\text { Proportion of births } \\
\text { among those under } 18 \\
\text { years (b) }\end{array}$ & $\begin{array}{l}\text { Percentage of all live births } \\
\text { which were deliveries } \\
\text { among women under } 18 \\
\text { years of age }\end{array}$ & Impact & $\checkmark$ & & & & & $\checkmark$ & $\checkmark$ & $\checkmark$ \\
\hline ARH & $\begin{array}{l}\text { Proportion of STIs among } \\
\text { those under } 18 \text { years (b, } \\
\text { d) }\end{array}$ & $\begin{array}{l}\text { Percentage of total number } \\
\text { of STIs diagnosed which } \\
\text { were diagnosed among } \\
\text { those under } 18 \text { years of age }\end{array}$ & Impact & $\checkmark$ & & & & & & & \\
\hline CAC & $\begin{array}{l}\text { Abortion services } \\
\text { performed with } \\
\text { appropriate technology } \\
\text { (b) }\end{array}$ & $\begin{array}{l}\text { Percentage of abortion } \\
\text { services performed in a } \\
\text { given period which are } \\
\text { performed with appropriate } \\
\text { technology (vacuum } \\
\text { aspiration or medical } \\
\text { methods) }\end{array}$ & Output & $\checkmark$ & & & & & & & \\
\hline CAC & $\begin{array}{l}\text { Awareness of legal } \\
\text { indications for } \\
\text { termination of } \\
\text { pregnancy (b) }\end{array}$ & $\begin{array}{l}\text { Percentage of providers } \\
\text { involved in abortion } \\
\text { services who are aware of } \\
\text { the legal indications for } \\
\text { termination of pregnancy }\end{array}$ & Output & & $\checkmark$ & & & & & & \\
\hline CAC & $\begin{array}{l}\text { Coverage of induced } \\
\text { abortion (b) }\end{array}$ & $\begin{array}{l}\text { Percentage of all women } \\
\text { receiving abortion services } \\
\text { in a given facility during a } \\
\text { given period who receive } \\
\text { induced procedures }\end{array}$ & Outcome & $\checkmark$ & & & & & & & \\
\hline CAC & $\begin{array}{l}\text { Coverage of post- } \\
\text { abortion contraception } \\
\text { (b) }\end{array}$ & $\begin{array}{l}\text { Percentage of all women } \\
\text { receiving abortion services } \\
\text { in a given facility during a } \\
\text { given period who receive } \\
\text { modern contraceptive } \\
\text { methods before leaving the } \\
\text { facility }\end{array}$ & Outcome & $\checkmark$ & & & & & & & \\
\hline FP & $\begin{array}{l}\text { Barriers to family } \\
\text { planning (e) }\end{array}$ & $\begin{array}{l}\text { Percentage of women not } \\
\text { currently using a family } \\
\text { planning method who } \\
\text { report at least one barrier } \\
\text { to family planning (except } \\
\text { for fertility-related reasons) }\end{array}$ & Output & & & $\checkmark$ & & & & & \\
\hline FP & $\begin{array}{l}\text { Difficulty accessing } \\
\text { contraception after the } \\
\text { disaster (c) }\end{array}$ & $\begin{array}{l}\text { Proportion of PP women } \\
\text { who have had difficulty } \\
\text { accessing their } \\
\text { contraceptive method since } \\
\text { the disaster }\end{array}$ & Output & & & $\checkmark$ & & & & & \\
\hline FP & $\begin{array}{l}\text { Community knowledge } \\
\text { concerning family } \\
\text { planning (b) }\end{array}$ & $\begin{array}{l}\text { Percentage of all sexually } \\
\text { active persons targeted for } \\
\text { family planning messages } \\
\text { who are able to cite major } \\
\text { messages about family } \\
\text { planning }\end{array}$ & Output & & & $\checkmark$ & & & & & \\
\hline
\end{tabular}


Table 2 Unique indicators identified ( $n=132)$ (Continued)

\begin{tabular}{|c|c|c|c|c|c|c|c|c|c|c|}
\hline \multirow[t]{2}{*}{ Domain } & \multirow{2}{*}{$\begin{array}{l}\text { Indicator (Sets it Appears } \\
\text { In) } \\
\text { [italics = appears in } \\
\text { multiple sets] }\end{array}$} & \multirow[t]{2}{*}{ Definition (Sets) } & \multirow[t]{2}{*}{ Type } & \multicolumn{5}{|c|}{ Data Source } & \multicolumn{2}{|c|}{$\begin{array}{l}\text { Overlap with } \\
\text { Priority } \\
\text { Indicators }\end{array}$} \\
\hline & & & & Facilities & $\begin{array}{l}\text { Service } \\
\text { Providers }\end{array}$ & $\begin{array}{l}\text { Affected } \\
\text { Population }\end{array}$ & $\begin{array}{l}\text { Program } \\
\text { Records }\end{array}$ & $\begin{array}{l}\text { Not } \\
\text { Specified }\end{array}$ & SDG GS & $\begin{array}{l}\text { Core } \\
\text { WHO }\end{array}$ \\
\hline FP & $\begin{array}{l}\text { Ever heard of modern } \\
\text { family planning methods } \\
\text { (e) }\end{array}$ & $\begin{array}{l}\text { Percentage of women of } \\
\text { reproductive age who have } \\
\text { ever heard of at least one } \\
\text { modern family planning } \\
\text { method }\end{array}$ & Output & & & $\checkmark$ & & & & \\
\hline FP & Contraceptive supply (b) & $\begin{array}{l}\text { Percentage of service } \\
\text { delivery points which } \\
\text { maintain a minimum of } 3 \\
\text { months' supply of } \\
\text { combined oral } \\
\text { contraceptive pills, } \\
\text { progestin-only pills, and } \\
\text { injectables }\end{array}$ & Process & $\checkmark$ & & & & & & \\
\hline FP & $\begin{array}{l}\text { Couple years protection } \\
\text { (d) }\end{array}$ & $\begin{array}{l}\text { Duration of contraceptive } \\
\text { protection provided by all } \\
\text { contraceptives sold or } \\
\text { distributed free of charge to } \\
\text { clients per 1-year period }\end{array}$ & Output & $\checkmark$ & & & & & & \\
\hline FP & $\begin{array}{l}\text { Coverage of FP } \\
\text { counselling (b) }\end{array}$ & $\begin{array}{l}\text { Percentage of clients } \\
\text { attending FP services who } \\
\text { are offered FP counselling } \\
\text { in addition to receiving a } \\
\text { method of contraception }\end{array}$ & Output & $\checkmark$ & & & & & & \\
\hline FP & $\begin{array}{l}\text { Unmet need for family } \\
\text { planning (e) }\end{array}$ & $\begin{array}{l}\text { Percentage of all women of } \\
\text { reproductive age who are } \\
\text { currently not using a family } \\
\text { planning method and are } \\
\text { at risk for pregnancy (not } \\
\text { using a method, not } \\
\text { currently pregnant or } \\
\text { postpartum, fecund, } \\
\text { sexually active in the last } \\
30 \text { days, and do not want a } \\
\text { baby in the immediate } \\
\text { future) }\end{array}$ & Outcome & & & $\checkmark$ & & & & \\
\hline FP & $\begin{array}{l}\text { Contraceptive prevalence } \\
(b, d, c)\end{array}$ & $\begin{array}{l}\text { Percentage of women 15- } \\
49 \text { years of age who are } \\
\text { using (or whose partner is } \\
\text { using) a contraceptive } \\
\text { method (b, d); Proportion of } \\
\text { PP women who are } \\
\text { currently practicing family } \\
\text { planning with their partner, } \\
\text { including natural family } \\
\text { planning methods (c) }\end{array}$ & Outcome & $\checkmark$ & & $\checkmark$ & & & & $\checkmark$ \\
\hline FP & $\begin{array}{l}\text { Modern contraceptive } \\
\text { prevalence (e) }\end{array}$ & $\begin{array}{l}\text { Percentage of all women of } \\
\text { reproductive age who are } \\
\text { using any modern family } \\
\text { planning method }\end{array}$ & Outcome & & & $\checkmark$ & & & & $\checkmark$ \\
\hline FP & $\begin{array}{l}\text { Use of family planning } \\
\text { before disaster (c) }\end{array}$ & $\begin{array}{l}\text { Proportion of PP women } \\
\text { who were using a } \\
\text { contraceptive method just } \\
\text { before the disaster }\end{array}$ & Outcome & & & $\checkmark$ & & & & \\
\hline FP & $\begin{array}{l}\text { Ever used modern family } \\
\text { planning methods (e) }\end{array}$ & $\begin{array}{l}\text { Percentage of women of } \\
\text { reproductive age who have } \\
\text { ever used at least one } \\
\text { modern family planning } \\
\text { method }\end{array}$ & Outcome & & & $\checkmark$ & & & & \\
\hline FP & Future intent to use a & Percentage of women not & Impact & & & $\checkmark$ & & & & \\
\hline
\end{tabular}


Table 2 Unique indicators identified ( $n=132)$ (Continued)

\begin{tabular}{|c|c|c|c|c|c|c|c|c|c|c|c|}
\hline \multirow[t]{2}{*}{ Domain } & \multirow{2}{*}{$\begin{array}{l}\text { Indicator (Sets it Appears } \\
\text { In) } \\
\text { [italics = appears in } \\
\text { multiple sets }]\end{array}$} & \multirow[t]{2}{*}{ Definition (Sets) } & \multirow[t]{2}{*}{ Type } & \multicolumn{5}{|c|}{ Data Source } & \multicolumn{3}{|c|}{$\begin{array}{l}\text { Overlap with } \\
\text { Priority } \\
\text { Indicators }\end{array}$} \\
\hline & & & & Facilities & $\begin{array}{l}\text { Service } \\
\text { Providers }\end{array}$ & $\begin{array}{l}\text { Affected } \\
\text { Population }\end{array}$ & $\begin{array}{l}\text { Program } \\
\text { Records }\end{array}$ & $\begin{array}{l}\text { Not } \\
\text { Specified }\end{array}$ & SDG & GS & $\begin{array}{l}\text { Core } \\
\text { WHO }\end{array}$ \\
\hline & $\begin{array}{l}\text { method in the next } 12 \\
\text { months (e) }\end{array}$ & $\begin{array}{l}\text { currently using a family } \\
\text { planning method who } \\
\text { intend to use a family } \\
\text { planning method in the } \\
\text { next } 12 \text { months }\end{array}$ & & & & & & & & & \\
\hline GBV & $\begin{array}{l}\text { Availability of clinical } \\
\text { management of rape } \\
\text { survivors }(a, h, f)\end{array}$ & $\begin{array}{l}\text { Percentage of health } \\
\text { facilities offering clinical } \\
\text { management of rape } \\
\text { survivors, including EC, PEP } \\
\text { and presumptive STI } \\
\text { treatment (a,h); Number of } \\
\text { functional health facilities } \\
\text { with clinical management } \\
\text { of rape survivors in a } \\
\text { defined administrative or } \\
\text { health area at a certain time } \\
\text { point (h); Not specified ( } f \text { ) }\end{array}$ & Output & $\checkmark$ & & & & & & $\checkmark$ & \\
\hline GBV & $\begin{array}{l}\text { Percentage of health } \\
\text { workers trained on } \\
\text { clinical management of } \\
\text { rape }(\mathrm{h})\end{array}$ & $\begin{array}{l}\text { Percentage of all health } \\
\text { workers which have been } \\
\text { trained on Clinical } \\
\text { Management of Rape }\end{array}$ & Output & & & & & $\checkmark$ & & & \\
\hline GBV & $\begin{array}{l}\text { Proportion of } \\
\text { community-based } \\
\text { workers trained in } \\
\text { psychosocial support for } \\
\text { GBV survivors (h) }\end{array}$ & $\begin{array}{l}\text { Percentage of all } \\
\text { community workers that } \\
\text { have been trained in GBV } \\
\text { psychosocial support }\end{array}$ & Output & & & & & $\checkmark$ & & & \\
\hline GBV & $\begin{array}{l}\text { Favorable to the } \\
\text { continuation of FGC (e) }\end{array}$ & $\begin{array}{l}\text { Percentage of women who } \\
\text { have ever heard of FGC } \\
\text { who think the practice } \\
\text { should continue }\end{array}$ & Outcome & & & $\checkmark$ & & & & & \\
\hline GBV & Future intent of FGC (e) & $\begin{array}{l}\text { Percentage of women who } \\
\text { have at least one daughter } \\
\text { and have heard of FGC } \\
\text { who intend to have } \\
\text { youngest daughter's } \\
\text { genitals cut in the future }\end{array}$ & Outcome & & & $\checkmark$ & & & & & \\
\hline GBV & $\begin{array}{l}\text { Prevalence of female } \\
\text { genital cutting (e) }\end{array}$ & $\begin{array}{l}\text { Percentage of women of } \\
\text { reproductive age who have } \\
\text { ever had their genitals cut }\end{array}$ & Outcome & & & $\checkmark$ & & & $\checkmark$ & $\checkmark$ & \\
\hline GBV & $\begin{array}{l}\text { Prevalence of female } \\
\text { genital cutting among } \\
\text { youngest daughter that } \\
\text { occurred in current } \\
\text { setting (e) }\end{array}$ & $\begin{array}{l}\text { Percentage of women who } \\
\text { have at least one daughter } \\
\text { and have heard of FGC } \\
\text { whose youngest daughter } \\
\text { ever had their genitals cut } \\
\text { and the cutting was done } \\
\text { in their current location }\end{array}$ & Outcome & & & $\checkmark$ & & & & & \\
\hline GBV & $\begin{array}{l}\text { Number of reported rape } \\
\text { cases }(a, d)\end{array}$ & $\begin{array}{l}\text { Number of rape cases } \\
\text { reported to health facilities } \\
\text { within a specific time } \\
\text { period (time period for } \\
\text { reporting to be set locally) } \\
\text { (a); Number of rape cases } \\
\text { reported per 10,000 } \\
\text { population per year (d) }\end{array}$ & Impact & $\checkmark$ & & & & & & & \\
\hline GBV & $\begin{array}{l}\text { Number of reported } \\
\text { sexual violence cases (b, } \\
\text { h) }\end{array}$ & $\begin{array}{l}\text { Number of cases of sexual } \\
\text { violence reported to health } \\
\text { services per month per 10, } \\
000 \text { population }\end{array}$ & Impact & $\checkmark$ & & & & & & & \\
\hline
\end{tabular}


Table 2 Unique indicators identified ( $n=132$ ) (Continued)

\begin{tabular}{|c|c|c|c|c|c|c|c|c|c|c|c|}
\hline \multirow[t]{2}{*}{ Domain } & \multirow{2}{*}{$\begin{array}{l}\text { Indicator (Sets it Appears } \\
\text { In) } \\
{[\text { italics = appears in }} \\
\text { multiple sets] }\end{array}$} & \multirow[t]{2}{*}{ Definition (Sets) } & \multirow[t]{2}{*}{ Type } & \multicolumn{5}{|c|}{ Data Source } & \multicolumn{3}{|c|}{$\begin{array}{l}\text { Overlap with } \\
\text { Priority } \\
\text { Indicators }\end{array}$} \\
\hline & & & & Facilities & $\begin{array}{l}\text { Service } \\
\text { Providers }\end{array}$ & $\begin{array}{l}\text { Affected } \\
\text { Population }\end{array}$ & $\begin{array}{l}\text { Program } \\
\text { Records }\end{array}$ & $\begin{array}{l}\text { Not } \\
\text { Specified }\end{array}$ & SDG & GS & $\begin{array}{l}\text { Core } \\
\text { WHO }\end{array}$ \\
\hline GBV & $\begin{array}{l}\text { Communities indicating } \\
\text { there is a risk of physical } \\
\text { or sexual violence (h) }\end{array}$ & $\begin{array}{l}\text { Percentage of all } \\
\text { communities which indicate } \\
\text { that there is a risk of } \\
\text { physical or sexual violence }\end{array}$ & Outcome & & & & & $\checkmark$ & & & \\
\hline GBV & $\begin{array}{l}\text { Intimate partner violence } \\
\text { (IPV) ever (e) }\end{array}$ & $\begin{array}{l}\text { Percentage of ever- } \\
\text { partnered women who } \\
\text { have ever experienced IPV } \\
\text { by a current or previous } \\
\text { partner }\end{array}$ & Impact & & & $\checkmark$ & & & & & \\
\hline GBV & IPV in past year (e) & $\begin{array}{l}\text { Percentage of women } \\
\text { partnered in the past } 12 \\
\text { months who have } \\
\text { experienced IPV in the past } \\
12 \text { months }\end{array}$ & Impact & & & $\checkmark$ & & & $\checkmark$ & $\checkmark$ & $\checkmark$ \\
\hline GBV & $\begin{array}{l}\text { Outsider physical } \\
\text { violence during conflict } \\
\text { (e) }\end{array}$ & $\begin{array}{l}\text { Percentage of women of } \\
\text { reproductive age who have } \\
\text { experienced physical } \\
\text { violence by someone } \\
\text { outside of their family } \\
\text { during the conflict }\end{array}$ & Impact & & & $\checkmark$ & & & & & \\
\hline GBV & $\begin{array}{l}\text { Outsider physical } \\
\text { violence post-conflict (e) }\end{array}$ & $\begin{array}{l}\text { Percentage of women of } \\
\text { reproductive age who have } \\
\text { experienced physical } \\
\text { violence by someone } \\
\text { outside of their family post- } \\
\text { conflict }\end{array}$ & Impact & & & $\checkmark$ & & & & & \\
\hline GBV & $\begin{array}{l}\text { Outsider sexual violence } \\
\text { during conflict (e) }\end{array}$ & $\begin{array}{l}\text { Percentage of women of } \\
\text { reproductive age who have } \\
\text { experienced sexual violence } \\
\text { by someone outside of } \\
\text { their family during the } \\
\text { conflict }\end{array}$ & Impact & & & $\checkmark$ & & & & & \\
\hline GBV & $\begin{array}{l}\text { Outsider sexual violence } \\
\text { post-conflict (e) }\end{array}$ & $\begin{array}{l}\text { Percentage of women of } \\
\text { reproductive age who have } \\
\text { experience sexual violence } \\
\text { by someone outside of } \\
\text { their family post-conflict }\end{array}$ & Impact & & & $\checkmark$ & & & & & \\
\hline GBV & $\begin{array}{l}\text { Physical intimate partner } \\
\text { violence since disaster (c) }\end{array}$ & $\begin{array}{l}\text { Percentage of pregnant } \\
\text { women reporting physical } \\
\text { violence by husband or } \\
\text { partner since the disaster }\end{array}$ & Impact & & & $\checkmark$ & & & & & \\
\hline GBV & $\begin{array}{l}\text { Physical violence by } \\
\text { family members in past } \\
\text { year (e) }\end{array}$ & $\begin{array}{l}\text { Percentage of women of } \\
\text { reproductive age who have } \\
\text { experienced physical } \\
\text { violence by family members } \\
\text { in the past year }\end{array}$ & Impact & & & $\checkmark$ & & & & & \\
\hline GBV & $\begin{array}{l}\text { Physical violence by } \\
\text { persons other than } \\
\text { intimate partners since } \\
\text { disaster (c) }\end{array}$ & $\begin{array}{l}\text { Percentage of pregnant } \\
\text { women reporting physical } \\
\text { violence by person other } \\
\text { than husband or partner } \\
\text { since the disaster }\end{array}$ & Impact & & & $\checkmark$ & & & & & \\
\hline GBV & $\begin{array}{l}\text { Sexual violence by } \\
\text { anyone, including } \\
\text { intimate partners since } \\
\text { disaster (c) }\end{array}$ & $\begin{array}{l}\text { Percentage of pregnant } \\
\text { women reporting sexual } \\
\text { violence by anyone } \\
\text { including husband or } \\
\text { partner since the disaster }\end{array}$ & Impact & & & $\checkmark$ & & & & & \\
\hline GBV & $\begin{array}{l}\text { Current needs for } \\
\text { services for family }\end{array}$ & $\begin{array}{l}\text { Percentage of pregnant } \\
\text { women reporting current }\end{array}$ & Outcome & & & $\checkmark$ & & & & & \\
\hline
\end{tabular}


Table 2 Unique indicators identified ( $n=132)$ (Continued)

\begin{tabular}{|c|c|c|c|c|c|c|c|c|c|c|c|}
\hline \multirow[t]{2}{*}{ Domain } & \multirow{2}{*}{$\begin{array}{l}\text { Indicator (Sets it Appears } \\
\text { In) } \\
\text { [italics = appears in } \\
\text { multiple sets] }\end{array}$} & \multirow[t]{2}{*}{ Definition (Sets) } & \multirow[t]{2}{*}{ Type } & \multicolumn{5}{|c|}{ Data Source } & \multicolumn{3}{|c|}{$\begin{array}{l}\text { Overlap with } \\
\text { Priority } \\
\text { Indicators }\end{array}$} \\
\hline & & & & Facilities & $\begin{array}{l}\text { Service } \\
\text { Providers }\end{array}$ & $\begin{array}{l}\text { Affected } \\
\text { Population }\end{array}$ & $\begin{array}{l}\text { Program } \\
\text { Records }\end{array}$ & $\begin{array}{l}\text { Not } \\
\text { Specified }\end{array}$ & SDG & GS & $\begin{array}{l}\text { Core } \\
\text { WHO }\end{array}$ \\
\hline & violence (c) & $\begin{array}{l}\text { need for services to reduce } \\
\text { violence in family }\end{array}$ & & & & & & & & & \\
\hline GBV & $\begin{array}{l}\text { Perceived effect of } \\
\text { violence on physical or } \\
\text { emotional health (c) }\end{array}$ & $\begin{array}{l}\text { Percentage of pregnant } \\
\text { women reporting perceived } \\
\text { effects of the violence on } \\
\text { physical or emotional } \\
\text { health }\end{array}$ & Impact & & & $\checkmark$ & & & & & \\
\hline GBV & $\begin{array}{l}\text { Sought treatment for } \\
\text { effects of violence (c) }\end{array}$ & $\begin{array}{l}\text { Percentage of pregnant } \\
\text { women who have } \\
\text { experienced violence since } \\
\text { the disaster and sought } \\
\text { treatment from a doctor, } \\
\text { counsellor, or any other } \\
\text { medical care provider for } \\
\text { resulting physical and/or } \\
\text { emotional problems }\end{array}$ & Impact & & & $\checkmark$ & & & & & \\
\hline GBV & $\begin{array}{l}\text { Humanitarian } \\
\text { organizations and service } \\
\text { providers with codes of } \\
\text { conduct for own staff (h) }\end{array}$ & $\begin{array}{l}\text { Percentage of humanitarian } \\
\text { organizations and service } \\
\text { providers that have in place } \\
\text { codes of conduct on } \\
\text { prevention of sexual } \\
\text { exploitation and abuse by } \\
\text { own staff }\end{array}$ & Output & & & & & $\checkmark$ & & & \\
\hline GBV & $\begin{array}{l}\text { Humanitarian } \\
\text { organizations and service } \\
\text { providers with } \\
\text { community-based } \\
\text { feedback and complaint } \\
\text { mechanisms (h) }\end{array}$ & $\begin{array}{l}\text { Percentage of humanitarian } \\
\text { organizations and service } \\
\text { providers that have in place } \\
\text { community-based feedback } \\
\text { and complaint mechanisms }\end{array}$ & Output & & & & & $\checkmark$ & & & \\
\hline GBV & Reporting IPV (e) & $\begin{array}{l}\text { Percentage of women who } \\
\text { have ever experienced IPV } \\
\text { who told an authority } \\
\text { (doctor/provider, police, } \\
\text { military, NGO worker) about } \\
\text { any incidence of IPV }\end{array}$ & Impact & & & $\checkmark$ & & & & & \\
\hline GBV & $\begin{array}{l}\text { Reporting outsider } \\
\text { violence (e) }\end{array}$ & $\begin{array}{l}\text { Percentage of women who } \\
\text { experienced outsider } \\
\text { violence during and post- } \\
\text { conflict who told an } \\
\text { authority (doctor/provider, } \\
\text { police, military, NGO } \\
\text { worker) about any incident } \\
\text { of outsider violence }\end{array}$ & Impact & & & $\checkmark$ & & & & & \\
\hline GBV & $\begin{array}{l}\text { Timing of EC provision ( } b \text {, } \\
d, h)\end{array}$ & $\begin{array}{l}\text { Percentage of all rape cases } \\
\text { reported within } 120 \mathrm{~h} \\
\text { where survivors receive ECP } \\
\text { within } 120 \mathrm{~h} \text { of incident (b, } \\
\mathrm{d}) \text {; Percentage of female } \\
\text { rape survivors who receive } \\
\text { ECP within } 120 \mathrm{~h} \text { of the } \\
\text { incident (h) }\end{array}$ & Outcome & $\checkmark$ & & & & & & & \\
\hline GBV & $\begin{array}{l}\text { Timing of PEP provision } \\
(b, d, h)\end{array}$ & $\begin{array}{l}\text { Percentage of all rape cases } \\
\text { reported within } 72 \mathrm{~h} \text { where } \\
\text { survivors receive PEP within } \\
72 \mathrm{~h} \text { of incident }(\mathrm{b}, \mathrm{d}) \text {; } \\
\text { Percentage of reported rape } \\
\text { cases where survivor } \\
\text { receives PEP for HIV within } \\
72 \mathrm{~h} \text { of incident ( } h \text { ) }\end{array}$ & Outcome & $\checkmark$ & & & & & & $\checkmark$ & \\
\hline
\end{tabular}


Table 2 Unique indicators identified ( $n=132)$ (Continued)

\begin{tabular}{|c|c|c|c|c|c|c|c|c|c|c|c|}
\hline \multirow[t]{2}{*}{ Domain } & \multirow{2}{*}{$\begin{array}{l}\text { Indicator (Sets it Appears } \\
\text { In) } \\
\text { [italics = appears in } \\
\text { multiple sets }]\end{array}$} & \multirow[t]{2}{*}{ Definition (Sets) } & \multirow[t]{2}{*}{ Type } & \multicolumn{5}{|c|}{ Data Source } & \multicolumn{3}{|c|}{$\begin{array}{l}\text { Overlap with } \\
\text { Priority } \\
\text { Indicators }\end{array}$} \\
\hline & & & & Facilities & $\begin{array}{l}\text { Service } \\
\text { Providers }\end{array}$ & $\begin{array}{l}\text { Affected } \\
\text { Population }\end{array}$ & $\begin{array}{l}\text { Program } \\
\text { Records }\end{array}$ & $\begin{array}{l}\text { Not } \\
\text { Specified }\end{array}$ & $\overline{S D G}$ & GS & $\begin{array}{l}\text { Core } \\
\text { WHO }\end{array}$ \\
\hline GBV & $\begin{array}{l}\text { Timing of STI prophylaxis } \\
(b, d)\end{array}$ & $\begin{array}{l}\text { Percentage of all rape cases } \\
\text { reported in which survivors } \\
\text { receive presumptive STI } \\
\text { treatment within } 2 \text { weeks of } \\
\text { an incident occurring (b); } \\
\text { Percentage of all rape cases } \\
\text { reported within } 2 \text { weeks } \\
\text { where survivors receive } \\
\text { presumptive STI treatment } \\
\text { within } 2 \text { weeks (d) }\end{array}$ & Outcome & $\checkmark$ & & & & & & & \\
\hline HIV & Eligibility for ART (d) & $\begin{array}{l}\text { Number of people enrolled } \\
\text { in HIV care and eligible for } \\
\text { ART but not started on ART } \\
\text { by end of period in one } \\
\text { camp over a one-year time } \\
\text { period }\end{array}$ & Impact & $\checkmark$ & & & & & & & \\
\hline HIV & Number on ART (d) & $\begin{array}{l}\text { Number of people started } \\
\text { on ART in one camp over a } \\
\text { one-month time period }\end{array}$ & Outcome & $\checkmark$ & & & & & & $\checkmark$ & $\checkmark$ \\
\hline HIV & $\begin{array}{l}\text { Coverage of HIV rapid } \\
\text { tests for safe blood } \\
\text { transfusion (a) }\end{array}$ & $\begin{array}{l}\text { Percentage of health service } \\
\text { delivery points with } \\
\text { sufficient HIV rapid tests to } \\
\text { screen blood for transfusion }\end{array}$ & Output & $\checkmark$ & & & & & & & \\
\hline HIV & $\begin{array}{l}\text { Quality of blood donation } \\
\text { screening }(b, d, f)\end{array}$ & $\begin{array}{l}\text { Percentage of donated } \\
\text { blood units screened for } \\
\text { HIV in a quality assured } \\
\text { manner (b, d); Not specified } \\
\text { (f) }\end{array}$ & Outcome & $\checkmark$ & & & & & & & \\
\hline HIV & $\begin{array}{l}\text { Condom distribution rate } \\
(d, a)\end{array}$ & $\begin{array}{l}\text { Number of condoms } \\
\text { distributed per person per } \\
\text { month (d); Number of male } \\
\text { condoms distributed per } \\
\text { total population per month } \\
\text { (a) }\end{array}$ & Output & $\checkmark$ & & & & & & & \\
\hline HIV & Condom use $(b, e)$ & $\begin{array}{l}\text { Percentage of sexually } \\
\text { active people reporting } \\
\text { condom use at last } \\
\text { intercourse (b); Percentage } \\
\text { of women who had sex } \\
\text { with a casual partner in the } \\
\text { last } 12 \text { months who did not } \\
\text { use a condom at last } \\
\text { intercourse } €\end{array}$ & Outcome & & & $\checkmark$ & & & & & $\checkmark$ \\
\hline HIV & $\begin{array}{l}\text { Accepting attitudes of } \\
\text { people living with HIV/ } \\
\text { AIDS (e) }\end{array}$ & $\begin{array}{l}\text { Percentage of women who } \\
\text { have ever heard of HIV/ } \\
\text { AIDS who indicate that } \\
\text { they: do not believe HIV } \\
\text { positive status of family } \\
\text { member should be kept } \\
\text { secret, are willing to care } \\
\text { for HIV positive family } \\
\text { member in home, believe } \\
\text { HIV positive teacher should } \\
\text { be allowed to continue } \\
\text { teaching, and would buy } \\
\text { fresh vegetables from an } \\
\text { HIV positive person }\end{array}$ & Outcome & & & $\checkmark$ & & & & & \\
\hline HIV & $\begin{array}{l}\text { Comprehensive correct } \\
\text { knowledge of HIV/AIDS }\end{array}$ & $\begin{array}{l}\text { Percentage of women of } \\
\text { reproductive age who }\end{array}$ & Outcome & & & $\checkmark$ & & & & & \\
\hline
\end{tabular}


Table 2 Unique indicators identified ( $n=132)$ (Continued)

\begin{tabular}{|c|c|c|c|c|c|c|c|c|c|c|}
\hline \multirow[t]{2}{*}{ Domain } & \multirow{2}{*}{$\begin{array}{l}\text { Indicator (Sets it Appears } \\
\text { In) } \\
\text { [italics = appears in } \\
\text { multiple sets] }\end{array}$} & \multirow[t]{2}{*}{ Definition (Sets) } & \multirow[t]{2}{*}{ Type } & \multicolumn{5}{|c|}{ Data Source } & \multicolumn{2}{|c|}{$\begin{array}{l}\text { Overlap with } \\
\text { Priority } \\
\text { Indicators }\end{array}$} \\
\hline & & & & Facilities & $\begin{array}{l}\text { Service } \\
\text { Providers }\end{array}$ & $\begin{array}{l}\text { Affected } \\
\text { Population }\end{array}$ & $\begin{array}{l}\text { Program } \\
\text { Records }\end{array}$ & $\begin{array}{l}\text { Not } \\
\text { Specified }\end{array}$ & SDG GS & $\begin{array}{l}\text { Core } \\
\text { WHO }\end{array}$ \\
\hline & (e) & $\begin{array}{l}\text { indicate that they: know } \\
\text { condoms prevent HIV, } \\
\text { know sex with only } 1 \\
\text { faithful uninfected partner } \\
\text { prevents HIV, do not think } \\
\text { mosquitoes transmit HIV, do } \\
\text { not think sharing food } \\
\text { transmits HIV, and know a } \\
\text { healthy-looking person can } \\
\text { have HIV }\end{array}$ & & & & & & & & \\
\hline HIV & $\begin{array}{l}\text { Comprehensive correct } \\
\text { knowledge of mother-to- } \\
\text { child transmission of } \\
\text { HIV/AIDS (e) }\end{array}$ & $\begin{array}{l}\text { Percentage of women of } \\
\text { reproductive age who } \\
\text { know that HIV/AIDS can be } \\
\text { transmitted from mother to } \\
\text { child during pregnancy or } \\
\text { delivery, and through } \\
\text { breastfeeding }\end{array}$ & Outcome & & & $\checkmark$ & & & & \\
\hline HIV & $\begin{array}{l}\text { Perceived risk of getting } \\
\text { HIV/AIDS (e) }\end{array}$ & $\begin{array}{l}\text { Percentage of women who } \\
\text { have ever heard of HIV/ } \\
\text { AIDS who believe they are } \\
\text { at moderate to high risk of } \\
\text { getting HIV/AIDS among } \\
\text { women who have ever } \\
\text { heard of HIV/AIDS }\end{array}$ & Outcome & & & $\checkmark$ & & & & \\
\hline HIV & $\begin{array}{l}\text { Enrolled in HIV care who } \\
\text { had TB status assessed } \\
\text { (d) }\end{array}$ & $\begin{array}{l}\text { Percentage of people } \\
\text { enrolled in HIV care and } \\
\text { seen for care who had TB } \\
\text { status assessed and } \\
\text { recorded during their last } \\
\text { visit }\end{array}$ & Output & $\checkmark$ & & & & & & \\
\hline HIV & $\begin{array}{l}\text { Targeting of people } \\
\text { most at risk of exposure } \\
\text { to HIV with a HIV } \\
\text { prevention programme } \\
\text { (f) }\end{array}$ & Not Specified & Output & & & & & $\checkmark$ & & \\
\hline HIV & Timing of PEP $(f, g)$ & $\begin{array}{l}\text { Percentage of individuals } \\
\text { potentially exposed to HIV } \\
\text { that receive PEP within } 72 \mathrm{~h} \\
\text { of incident }\end{array}$ & Outcome & & & & $\checkmark$ & & & \\
\hline HIV & $\begin{array}{l}\text { Infant HIV positive rate } \\
\text { (d) }\end{array}$ & $\begin{array}{l}\text { Percentage of all deliveries } \\
\text { to HIV-positive mothers } \\
\text { where the infant tested } \\
\text { positive at } 18 \text { months of } \\
\text { age }\end{array}$ & Impact & $\checkmark$ & & & & & & \\
\hline HIV & $\begin{array}{l}\text { PMTCT ARV coverage } \\
\text { (infant) (d) }\end{array}$ & $\begin{array}{l}\text { Percentage of all deliveries } \\
\text { to HIV-positive mothers } \\
\text { where the infant swallowed } \\
\text { ARV within } 72 \mathrm{~h} \text { of delivery }\end{array}$ & Outcome & $\checkmark$ & & & & & & \\
\hline HIV & $\begin{array}{l}\text { PMTCT ARV coverage } \\
\text { (mother) (d) }\end{array}$ & $\begin{array}{l}\text { Percentage of all deliveries } \\
\text { to HIV-positive mothers } \\
\text { where the mother } \\
\text { swallowed ARV during } \\
\text { labour/delivery }\end{array}$ & Outcome & $\checkmark$ & & & & & & $\checkmark$ \\
\hline HIV & $\begin{array}{l}\text { PMTCT ARV coverage } \\
\text { (mother-infant pair) }(b, d)\end{array}$ & $\begin{array}{l}\text { Percentage of all deliveries } \\
\text { to HIV-positive mothers } \\
\text { where the mother-infant } \\
\text { pair swallowed ARV } \\
\text { according to protocol }\end{array}$ & Outcome & $\checkmark$ & & & & & & $\checkmark$ \\
\hline
\end{tabular}


Table 2 Unique indicators identified ( $n=132)$ (Continued)

\begin{tabular}{|c|c|c|c|c|c|c|c|c|c|c|}
\hline \multirow[t]{2}{*}{ Domain } & \multirow{2}{*}{$\begin{array}{l}\text { Indicator (Sets it Appears } \\
\text { In) } \\
\text { [italics = appears in } \\
\text { multiple sets] }\end{array}$} & \multirow[t]{2}{*}{ Definition (Sets) } & \multirow[t]{2}{*}{ Type } & \multicolumn{5}{|c|}{ Data Source } & \multicolumn{2}{|c|}{$\begin{array}{l}\text { Overlap with } \\
\text { Priority } \\
\text { Indicators }\end{array}$} \\
\hline & & & & Facilities & $\begin{array}{l}\text { Service } \\
\text { Providers }\end{array}$ & $\begin{array}{l}\text { Affected } \\
\text { Population }\end{array}$ & $\begin{array}{l}\text { Program } \\
\text { Records }\end{array}$ & $\begin{array}{l}\text { Not } \\
\text { Specified }\end{array}$ & SDG GS & $\begin{array}{l}\text { Core } \\
\text { WHO }\end{array}$ \\
\hline HIV & $\begin{array}{l}\text { PMTCT ARV use for } \\
\text { treatment }(d)\end{array}$ & $\begin{array}{l}\text { Percentage of all eligible } \\
\text { HIV-infected pregnant } \\
\text { women who received anti- } \\
\text { retroviral as treatment }\end{array}$ & Outcome & $\checkmark$ & & & & & & \\
\hline HIV & $\begin{array}{l}\text { Receipt of ARV drugs for } \\
\text { PMTCT by pregnant } \\
\text { women known to be HIV } \\
\text { positive }(f)\end{array}$ & Not Specified & Outcome & & & & & $\checkmark$ & & $\checkmark$ \\
\hline HIV & $\begin{array}{l}\text { PMTCT Co-trimoxazole } \\
\text { prophylaxis (infant) (d) }\end{array}$ & $\begin{array}{l}\text { Percentage of all deliveries } \\
\text { to HIV-positive mothers } \\
\text { where the infant was } \\
\text { started on co-trimoxazole } \\
\text { prophylaxis }\end{array}$ & Outcome & $\checkmark$ & & & & & & \\
\hline HIV & $\begin{array}{l}\text { PMTCT Co-trimoxazole } \\
\text { prophylaxis (mother) (d) }\end{array}$ & $\begin{array}{l}\text { Percentage of all deliveries } \\
\text { to HIV-positive mothers } \\
\text { where the mother was } \\
\text { started on co-trimoxazole } \\
\text { prophylaxis }\end{array}$ & Outcome & $\checkmark$ & & & & & & \\
\hline HIV & $\begin{array}{l}\text { PMTCT pre-test } \\
\text { counselling coverage }(b, \\
\text { d) }\end{array}$ & $\begin{array}{l}\text { Percentage of first ANC visit } \\
\text { clients who were pre-test } \\
\text { counselled }\end{array}$ & Output & $\checkmark$ & & & & & & \\
\hline HIV & $\begin{array}{l}\text { PMTCT Exclusive } \\
\text { breastfeeding rate (d) }\end{array}$ & $\begin{array}{l}\text { Percentage of all deliveries } \\
\text { to HIV-positive mothers } \\
\text { where the mother plans to } \\
\text { exclusively breastfeed after } \\
\text { delivery }\end{array}$ & Outcome & $\checkmark$ & & & & & & \\
\hline HIV & $\begin{array}{l}\text { PMTCT Family planning } \\
\text { acceptance rate }(\mathrm{d})\end{array}$ & $\begin{array}{l}\text { Percentage of all deliveries } \\
\text { to HIV-positive mothers } \\
\text { where the mother accepted } \\
\text { a modern method of family } \\
\text { planning after delivery }\end{array}$ & Outcome & $\checkmark$ & & & & & & \\
\hline HIV & $\begin{array}{l}\text { PMTCT HIV prevalence } \\
\text { rate (d) }\end{array}$ & $\begin{array}{l}\text { Percentage of all first } \\
\text { antenatal care visits with } \\
\text { HIV testing where the } \\
\text { patient tested positive for } \\
\text { HIV }\end{array}$ & Impact & $\checkmark$ & & & & & & \\
\hline HIV & $\begin{array}{l}\text { PMTCT Home-based } \\
\text { counselling (d) }\end{array}$ & $\begin{array}{l}\text { Percentage of all deliveries } \\
\text { to HIV-positive mothers } \\
\text { where the mother received } \\
\text { at least one home-based } \\
\text { counselling visit after } \\
\text { delivery }\end{array}$ & Output & $\checkmark$ & & & & & & \\
\hline HIV & $\begin{array}{l}\text { PMTCT Post-test } \\
\text { counselling and result } \\
\text { (partners) (d) }\end{array}$ & $\begin{array}{l}\text { Percentage of all antenatal } \\
\text { care partners, who were } \\
\text { pre-test counselled and HIV } \\
\text { tested, who received post- } \\
\text { test results and counselling }\end{array}$ & Output & $\checkmark$ & & & & & & \\
\hline HIV & $\begin{array}{l}\text { PMTCT post-test } \\
\text { counselling and result ( } b \text {, } \\
\text { d) }\end{array}$ & $\begin{array}{l}\text { Percentage of first ANC visit } \\
\text { clients tested for HIV who } \\
\text { receive post-test result and } \\
\text { counselling }\end{array}$ & Output & $\checkmark$ & & & & & & \\
\hline HIV & $\begin{array}{l}\text { Proportional PMTCT } \\
\text { service use by Nationals } \\
\text { (d) }\end{array}$ & $\begin{array}{l}\text { Percentage of all PMTCT } \\
\text { clients pre-test counselled } \\
\text { who were Nationals }\end{array}$ & Outcome & $\checkmark$ & & & & & & \\
\hline HIV & $\begin{array}{l}\text { Coverage of supplies for } \\
\text { standard precautions ( } g \text {, } \\
\text { a) }\end{array}$ & $\begin{array}{l}\text { Percentage of health service } \\
\text { delivery points with } \\
\text { adequate supplies to carry }\end{array}$ & Output & $\checkmark$ & & & & & & \\
\hline
\end{tabular}


Table 2 Unique indicators identified ( $n=132)$ (Continued)

\begin{tabular}{|c|c|c|c|c|c|c|c|c|c|c|c|}
\hline \multirow[t]{2}{*}{ Domain } & \multirow{2}{*}{$\begin{array}{l}\text { Indicator (Sets it Appears } \\
\text { In) } \\
\text { [italics = appears in } \\
\text { multiple sets }]\end{array}$} & \multirow[t]{2}{*}{ Definition (Sets) } & \multirow[t]{2}{*}{ Type } & \multicolumn{5}{|c|}{ Data Source } & \multicolumn{3}{|c|}{$\begin{array}{l}\text { Overlap with } \\
\text { Priority } \\
\text { Indicators }\end{array}$} \\
\hline & & & & Facilities & $\begin{array}{l}\text { Service } \\
\text { Providers }\end{array}$ & $\begin{array}{l}\text { Affected } \\
\text { Population }\end{array}$ & $\begin{array}{l}\text { Program } \\
\text { Records }\end{array}$ & $\begin{array}{l}\text { Not } \\
\text { Specified }\end{array}$ & SDG & GS & $\begin{array}{l}\text { Core } \\
\text { WHO }\end{array}$ \\
\hline & & out standard precautions & & & & & & & & & \\
\hline HIV & $\begin{array}{l}\text { Proportional VCT service } \\
\text { use by nationals (d) }\end{array}$ & $\begin{array}{l}\text { Percentage of all VCT clients } \\
\text { pre-test counselled who are } \\
\text { Nationals }\end{array}$ & Outcome & $\checkmark$ & & & & & & & \\
\hline HIV & $\begin{array}{l}\text { Received HIV test results } \\
\text { in the last } 12 \text { months (e) }\end{array}$ & $\begin{array}{l}\text { Percentage of women who } \\
\text { were tested for HIV in the } \\
\text { last } 12 \text { months who } \\
\text { received their HIV test } \\
\text { results }\end{array}$ & Output & & & $\checkmark$ & & & & & \\
\hline HIV & $\begin{array}{l}\text { VCT post-test counselling } \\
\text { and result }(b, d)\end{array}$ & $\begin{array}{l}\text { Percentage of VCT clients } \\
\text { tested for HIV who received } \\
\text { post-test result and } \\
\text { counselling }\end{array}$ & Output & $\checkmark$ & & & & & & & \\
\hline HIV & $\begin{array}{l}\text { Would have an HIV test } \\
\text { in the future (e) }\end{array}$ & $\begin{array}{l}\text { Percentage of women who } \\
\text { have ever heard of HIV/ } \\
\text { AIDS who would go for an } \\
\text { HIV test in the future }\end{array}$ & Outcome & & & $\checkmark$ & & & & & \\
\hline $\mathrm{MH}$ & $\begin{array}{l}\text { Complete ANC coverage } \\
(b, d, e)\end{array}$ & $\begin{array}{l}\text { Percentage of total number } \\
\text { of live births in which the } \\
\text { mother made at least } 4 \\
\text { ANC visits during the } \\
\text { antenatal period at the time } \\
\text { of delivery (b, } d \text { ); } \\
\text { Percentage of all women } \\
\text { whose most recent } \\
\text { pregnancy ended in a live } \\
\text { birth or stillbirth in the last } \\
\text { two years who received at } \\
\text { least } 3 \text { antenatal care visits } \\
\text { by a trained provider (e) }\end{array}$ & Outcome & $\checkmark$ & & $\checkmark$ & & & & $\checkmark$ & $\checkmark$ \\
\hline $\mathrm{MH}$ & $\begin{array}{l}\text { Coverage of syphilis } \\
\text { screening }(b, d)\end{array}$ & $\begin{array}{l}\text { Percentage of total number } \\
\text { of live births where the } \\
\text { mother had been screened } \\
\text { for syphilis during the } \\
\text { antenatal period at the time } \\
\text { of delivery }\end{array}$ & Outcome & $\checkmark$ & & & & & & $\checkmark$ & \\
\hline $\mathrm{MH}$ & $\begin{array}{l}\text { Tetanus vaccination } \\
\text { coverage }(b, d)\end{array}$ & $\begin{array}{l}\text { Percentage of total number } \\
\text { of live births where the } \\
\text { mother had received } 2 \\
\text { doses of tetanus toxoid } \\
\text { vaccine (or were fully } \\
\text { vaccinated) during the } \\
\text { antenatal period at the time } \\
\text { of delivery }\end{array}$ & Outcome & $\checkmark$ & & & & & & & \\
\hline $\mathrm{MH}$ & $\begin{array}{l}\text { Timing of first antenatal } \\
\text { visit (d) }\end{array}$ & $\begin{array}{l}\text { Percentage of first-time } \\
\text { antenatal visits that were } \\
\text { made in the first trimester }\end{array}$ & Outcome & $\checkmark$ & & & & & & & \\
\hline $\mathrm{MH}$ & $\begin{array}{l}\text { Coverage of intermittent } \\
\text { presumptive treatment } \\
\text { for malaria (d) }\end{array}$ & $\begin{array}{l}\text { Percentage of all live births } \\
\text { where the mother had } \\
\text { received two doses of } \\
\text { fansidar prophylaxis during } \\
\text { the antenatal period at the } \\
\text { time of delivery }\end{array}$ & Outcome & $\checkmark$ & & & & & & & $\checkmark$ \\
\hline $\mathrm{MH}$ & $\begin{array}{l}\text { Coverage of long-lasting } \\
\text { insecticidal nets ( } \mathrm{g} \text { ) }\end{array}$ & $\begin{array}{l}\text { Number of pregnant and } \\
\text { lactating women and } \\
\text { children under } 5 \text { sleeping } \\
\text { nightly under net }\end{array}$ & Outcome & & & & $\checkmark$ & & & & \\
\hline $\mathrm{MH}$ & Percentage of caesarean & Percentage of live births & Outcome & $\checkmark$ & & & & & & & \\
\hline
\end{tabular}


Table 2 Unique indicators identified ( $n=132)$ (Continued)

\begin{tabular}{|c|c|c|c|c|c|c|c|c|c|c|c|}
\hline \multirow[t]{2}{*}{ Domain } & \multirow{2}{*}{$\begin{array}{l}\text { Indicator (Sets it Appears } \\
\text { In) } \\
\text { [italics = appears in } \\
\text { multiple sets }]\end{array}$} & \multirow[t]{2}{*}{ Definition (Sets) } & \multirow[t]{2}{*}{ Type } & \multicolumn{5}{|c|}{ Data Source } & \multicolumn{3}{|c|}{$\begin{array}{l}\text { Overlap with } \\
\text { Priority } \\
\text { Indicators }\end{array}$} \\
\hline & & & & Facilities & $\begin{array}{l}\text { Service } \\
\text { Providers }\end{array}$ & $\begin{array}{l}\text { Affected } \\
\text { Population }\end{array}$ & $\begin{array}{l}\text { Program } \\
\text { Records }\end{array}$ & $\begin{array}{l}\text { Not } \\
\text { Specified }\end{array}$ & SDG & GS & $\begin{array}{l}\text { Core } \\
\text { WHO }\end{array}$ \\
\hline & $\operatorname{section}(b, d, f, h)$ & $\begin{array}{l}\text { which are delivered via } \\
\text { Caesarean sections }\end{array}$ & & & & & & & & & \\
\hline $\mathrm{MH}$ & $\begin{array}{l}\text { Coverage of clean delivery } \\
\text { kits }(a, g, f)\end{array}$ & $\begin{array}{l}\text { Number of clean delivery } \\
\text { kits distributed per hundred } \\
\text { pregnant women per } \\
\text { month }(a, g) \text {; Women in } \\
\text { third trimester of pregnancy } \\
\text { who have received clean } \\
\text { delivery kits (f) }\end{array}$ & Output & & & & $\checkmark$ & & & & \\
\hline $\mathrm{MH}$ & $\begin{array}{l}\text { Delivery assisted by a } \\
\text { skilled attendant }(b, d, h \text {, } \\
\text { e) }\end{array}$ & $\begin{array}{l}\text { Percentage of all deliveries } \\
\text { which are attended by a } \\
\text { trained health worker }(b) \text {; } \\
\text { Percentage of all live births } \\
\text { attended by skilled birth } \\
\text { attendants }(d, h) ; \\
\text { Percentage of all women } \\
\text { whose most recent } \\
\text { pregnancy ended in a live } \\
\text { birth or stillbirth in the last } \\
\text { two years whose delivery } \\
\text { was attended by a trained } \\
\text { health care provider at a } \\
\text { health facility (e) }\end{array}$ & Outcome & $\checkmark$ & & $\checkmark$ & & & $\checkmark$ & $\checkmark$ & $\checkmark$ \\
\hline $\mathrm{MH}$ & $\begin{array}{l}\text { BEmOC services } \\
\text { availability }(f, h)\end{array}$ & $\begin{array}{l}\text { Number of functional } \\
\text { health facilities with BEmOC } \\
\text { per } 500,000 \text { population }\end{array}$ & Output & $\checkmark$ & & & & & & $\checkmark$ & \\
\hline $\mathrm{MH}$ & $\begin{array}{l}\text { CEmOC services } \\
\text { availability }(f, h)\end{array}$ & $\begin{array}{l}\text { Number of functional } \\
\text { health facilities with CEmOC } \\
\text { per } 500,000 \text { population }\end{array}$ & Output & $\checkmark$ & & & & & & $\checkmark$ & \\
\hline $\mathrm{MH}$ & EmOC needs met (b) & $\begin{array}{l}\text { Percentage of all deliveries } \\
\text { with major obstetric } \\
\text { complications which are } \\
\text { treated at an EmOC facility }\end{array}$ & Outcome & $\checkmark$ & & & & & & & \\
\hline $\mathrm{MH}$ & $\begin{array}{l}\text { EmOC services } \\
\text { availability (b) }\end{array}$ & $\begin{array}{l}\text { Number of health facilities } \\
\text { with basic and/or } \\
\text { comprehensive obstetric } \\
\text { care per } 500,000 \text { population } \\
\text { by administrative unit }\end{array}$ & Output & $\checkmark$ & & & & & & $\checkmark$ & \\
\hline $\mathrm{MH}$ & $\begin{array}{l}\text { EmOC services utilization } \\
(b, d)\end{array}$ & $\begin{array}{l}\text { Percentage of all deliveries } \\
\text { which occur in an EmOC } \\
\text { center }\end{array}$ & Outcome & $\checkmark$ & & & & & & & \\
\hline $\mathrm{MH}$ & $\begin{array}{l}\text { Health problems during } \\
\text { pregnancy (c) }\end{array}$ & $\begin{array}{l}\text { Percentage of women } \\
\text { reporting health problems } \\
\text { that require ongoing care. } \\
\text { This includes diabetes, } \\
\text { vaginal bleeding, urinary } \\
\text { tract infections, sever } \\
\text { nausea and vomiting, } \\
\text { hypertensive disorders, } \\
\text { heart problems, and any } \\
\text { other identified by the } \\
\text { interviewee. }\end{array}$ & Outcome & & & $\checkmark$ & & & & & \\
\hline $\mathrm{MH}$ & $\begin{array}{l}\text { Prevalence of anaemia } \\
\text { (d) }\end{array}$ & $\begin{array}{l}\text { Percentage of all antenatal } \\
\text { mothers tested for anaemia } \\
\text { with severe and moderate } \\
\text { anaemia }\end{array}$ & Impact & $\checkmark$ & & & & & & & \\
\hline $\mathrm{MH}$ & Prevalence of syphilis (d) & $\begin{array}{l}\text { Percentage of antenatal } \\
\text { mothers tested for syphilis }\end{array}$ & Impact & $\checkmark$ & & & & & & & \\
\hline
\end{tabular}


Table 2 Unique indicators identified ( $n=132)$ (Continued)

\begin{tabular}{|c|c|c|c|c|c|c|c|c|c|c|c|}
\hline \multirow[t]{2}{*}{ Domain } & \multirow{2}{*}{$\begin{array}{l}\text { Indicator (Sets it Appears } \\
\text { In) } \\
\text { [italics = appears in } \\
\text { multiple sets }]\end{array}$} & \multirow[t]{2}{*}{ Definition (Sets) } & \multirow[t]{2}{*}{ Type } & \multicolumn{5}{|c|}{ Data Source } & \multicolumn{3}{|c|}{$\begin{array}{l}\text { Overlap with } \\
\text { Priority } \\
\text { Indicators }\end{array}$} \\
\hline & & & & Facilities & $\begin{array}{l}\text { Service } \\
\text { Providers }\end{array}$ & $\begin{array}{l}\text { Affected } \\
\text { Population }\end{array}$ & $\begin{array}{l}\text { Program } \\
\text { Records }\end{array}$ & $\begin{array}{l}\text { Not } \\
\text { Specified }\end{array}$ & SDG & GS & $\begin{array}{l}\text { Core } \\
\text { WHO }\end{array}$ \\
\hline & & $\begin{array}{l}\text { that tested positive on } \\
\text { Rapid Plasma Reagent } \\
\text { testing }\end{array}$ & & & & & & & & & \\
\hline $\mathrm{MH}$ & $\begin{array}{l}\text { Help-seeking behavior } \\
\text { for postpartum } \\
\text { complications (e) }\end{array}$ & $\begin{array}{l}\text { Percentage of all women } \\
\text { who reported postpartum } \\
\text { complications after their } \\
\text { most recent pregnancy that } \\
\text { ended in a live birth or } \\
\text { stillbirth in the last two } \\
\text { years who sought help at a } \\
\text { health facility }\end{array}$ & Outcome & & & $\checkmark$ & & & & & \\
\hline $\mathrm{MH}$ & $\begin{array}{l}\text { Help-seeking behavior } \\
\text { for pregnancy } \\
\text { complications (e) }\end{array}$ & $\begin{array}{l}\text { Percentage of all women } \\
\text { who reported complications } \\
\text { before labor or delivery } \\
\text { with their most recent } \\
\text { pregnancy that ended in a } \\
\text { live birth or stillbirth in the } \\
\text { last two years who sought } \\
\text { help at a health facility }\end{array}$ & Outcome & & & $\checkmark$ & & & & & \\
\hline $\mathrm{MH}$ & $\begin{array}{l}\text { Knowledge of danger } \\
\text { signs of pregnancy } \\
\text { complications (e) }\end{array}$ & $\begin{array}{l}\text { Percentage of all women of } \\
\text { reproductive age who } \\
\text { know at least two danger } \\
\text { signs of pregnancy } \\
\text { complications }\end{array}$ & Output & & & $\checkmark$ & & & & & \\
\hline $\mathrm{MH}$ & $\begin{array}{l}\text { Pregnant women aware } \\
\text { of where to go for } \\
\text { labour and delivery (g) }\end{array}$ & $\begin{array}{l}\text { Percentage of women who } \\
\text { receive birth kits who } \\
\text { receive counselling on the } \\
\text { need for skilled birth } \\
\text { attendance for delivery and } \\
\text { know where to go }\end{array}$ & Output & & & $\checkmark$ & & & & & \\
\hline $\mathrm{MH}$ & $\begin{array}{l}\text { Proportion of low birth } \\
\text { weight }(c, b, d)\end{array}$ & $\begin{array}{l}\text { Proportion of postpartum } \\
\text { women who reported their } \\
\text { infants weighed less than } \\
2500 \mathrm{~g} \text { at birth (c); } \\
\text { Percentage of total number } \\
\text { of live births (with birth } \\
\text { weight recorded) where the } \\
\text { infant weighs less than } \\
2500 \mathrm{~g}(\mathrm{~b}, \mathrm{~d})\end{array}$ & Impact & $\checkmark$ & & $\checkmark$ & & & & & $\checkmark$ \\
\hline $\mathrm{MH}$ & Preterm birth (c) & $\begin{array}{l}\text { Proportion of PP women } \\
\text { who reported they } \\
\text { delivered a live singleton } \\
\text { baby at least three weeks } \\
\text { before their due date }\end{array}$ & Impact & & & $\checkmark$ & & & & & \\
\hline $\mathrm{MH}$ & $\begin{array}{l}\text { Direct obstetric case } \\
\text { fatality rate }(b)\end{array}$ & $\begin{array}{l}\text { Percentage of all women } \\
\text { seen for a direct obstetric } \\
\text { complication at an EmOC } \\
\text { facility who die of a direct } \\
\text { obstetric complication }\end{array}$ & Impact & $\checkmark$ & & & & & & & \\
\hline $\mathrm{MH}$ & $\begin{array}{l}\text { Investigation of maternal } \\
\text { deaths }(b, d)\end{array}$ & $\begin{array}{l}\text { Percentage of total number } \\
\text { of reported maternal deaths } \\
\text { which are investigated }\end{array}$ & Output & $\checkmark$ & & & & & & & $\checkmark$ \\
\hline $\mathrm{MH}$ & $\begin{array}{l}\text { Maternal mortality ratio } \\
\text { (d) }\end{array}$ & $\begin{array}{l}\text { Number of pregnancy- } \\
\text { related deaths per 100,000 } \\
\text { live births }\end{array}$ & Impact & $\checkmark$ & & & & & $\checkmark$ & $\checkmark$ & $\checkmark$ \\
\hline $\mathrm{MH}$ & $\begin{array}{l}\text { Number of maternal } \\
\text { deaths reported (d) }\end{array}$ & $\begin{array}{l}\text { Number of reported } \\
\text { maternal deaths }\end{array}$ & Output & $\checkmark$ & & & & & & & \\
\hline $\mathrm{MH}$ & Abortion ratio (d) & Number of abortions & Impact & $\checkmark$ & & & & & & & \\
\hline
\end{tabular}


Table 2 Unique indicators identified ( $n=132$ ) (Continued)

\begin{tabular}{|c|c|c|c|c|c|c|c|c|c|c|c|}
\hline \multirow[t]{2}{*}{ Domain } & \multirow{2}{*}{$\begin{array}{l}\text { Indicator (Sets it Appears } \\
\text { In) } \\
\text { [italics = appears in } \\
\text { multiple sets] }\end{array}$} & \multirow[t]{2}{*}{ Definition (Sets) } & \multirow[t]{2}{*}{ Type } & \multicolumn{5}{|c|}{ Data Source } & \multicolumn{3}{|c|}{$\begin{array}{l}\text { Overlap with } \\
\text { Priority } \\
\text { Indicators }\end{array}$} \\
\hline & & & & Facilities & $\begin{array}{l}\text { Service } \\
\text { Providers }\end{array}$ & $\begin{array}{l}\text { Affected } \\
\text { Population }\end{array}$ & $\begin{array}{l}\text { Program } \\
\text { Records }\end{array}$ & $\begin{array}{l}\text { Not } \\
\text { Specified }\end{array}$ & SDG & GS & $\begin{array}{l}\text { Core } \\
\text { WHO }\end{array}$ \\
\hline & & $\begin{array}{l}\text { (defined as spontaneous } \\
\text { miscarriage before } 22 \text { weeks } \\
\text { gestation) per } 1000 \text { live } \\
\text { births }\end{array}$ & & & & & & & & & \\
\hline $\mathrm{MH}$ & $\begin{array}{l}\text { Neonatal mortality rate } \\
(b, d)\end{array}$ & $\begin{array}{l}\text { Number of live born infants } \\
\text { who die at less than } 28 \\
\text { days of age per } 1000 \text { live } \\
\text { births in the specified } \\
\text { period }\end{array}$ & Impact & $\checkmark$ & & & & & $\checkmark$ & $\checkmark$ & $\checkmark$ \\
\hline $\mathrm{MH}$ & Stillbirth rate $(b, d)$ & $\begin{array}{l}\text { Number of still births per } \\
1000 \text { births (still and live) } \\
\text { per month }\end{array}$ & Impact & $\checkmark$ & & & & & & $\checkmark$ & $\checkmark$ \\
\hline $\mathrm{MH}$ & $\begin{array}{l}\text { Access to postpartum } \\
\text { care (c) }\end{array}$ & Not specified & Output & & & $\checkmark$ & & & & & \\
\hline $\mathrm{MH}$ & $\begin{array}{l}\text { Postnatal care coverage } \\
(b, d, e)\end{array}$ & $\begin{array}{l}\text { Percentage of all live births } \\
\text { where the mother received } \\
\text { postnatal care } 3 \text { times } \\
\text { within } 6 \text { weeks of delivery } \\
\text { (b, d); Percentage of all } \\
\text { women whose most recent } \\
\text { pregnancy ended in a live } \\
\text { birth or stillbirth in the last } \\
\text { two years who received at } \\
\text { least } 1 \text { postpartum care visit } \\
\text { within six weeks after } \\
\text { delivery (e) }\end{array}$ & Outcome & $\checkmark$ & & $\checkmark$ & & & & $\checkmark$ & $\checkmark$ \\
\hline $\mathrm{MH}$ & $\begin{array}{l}\text { Disaster related difficulty } \\
\text { when accessing } \\
\text { postpartum care (c) }\end{array}$ & $\begin{array}{l}\text { Percentage of women who } \\
\text { experienced difficulty } \\
\text { obtaining a postpartum } \\
\text { check-up because of the } \\
\text { disaster }\end{array}$ & Outcome & & & $\checkmark$ & & & & & \\
\hline $\mathrm{MH}$ & Crude birth rate $(\mathrm{d})$ & $\begin{array}{l}\text { Number of live births per } \\
1000 \text { total population }\end{array}$ & Impact & $\checkmark$ & & & & & & & \\
\hline $\mathrm{MH}$ & Currently pregnant (e) & $\begin{array}{l}\text { Percentage of all women of } \\
\text { reproductive age who are } \\
\text { currently pregnant }\end{array}$ & Impact & & & $\checkmark$ & & & & & \\
\hline $\mathrm{MH}$ & $\begin{array}{l}\text { Pregnancies in last two } \\
\text { years (e) }\end{array}$ & $\begin{array}{l}\text { Percentage of all women of } \\
\text { reproductive age who have } \\
\text { had one or more } \\
\text { pregnancies in the last two } \\
\text { years }\end{array}$ & Impact & & & $\checkmark$ & & & & & \\
\hline STI & $\begin{array}{l}\text { Incidence of genital ulcer } \\
\text { disease }(b, d)\end{array}$ & $\begin{array}{l}\text { Number of cases of genital } \\
\text { ulcer disease per } 1000 \\
\text { population per month }\end{array}$ & Impact & $\checkmark$ & & & & & & & $\checkmark$ \\
\hline STI & $\begin{array}{l}\text { Incidence of male urethral } \\
\text { discharge }(b, d)\end{array}$ & $\begin{array}{l}\text { Number of cases of male } \\
\text { urethral discharge reported } \\
\text { per } 1000 \text { population per } \\
\text { month }\end{array}$ & Impact & $\checkmark$ & & & & & & & $\checkmark$ \\
\hline STI & $\begin{array}{l}\text { Selected STI-associated } \\
\text { symptoms in the past } \\
12 \text { months (e) }\end{array}$ & $\begin{array}{l}\text { Percentage of women of } \\
\text { reproductive age who have } \\
\text { had unusual genital } \\
\text { discharge and/or genital } \\
\text { ulcers or sores in the last } \\
12 \text { months }\end{array}$ & Impact & & & $\checkmark$ & & & & & $\checkmark$ \\
\hline STI & $\begin{array}{l}\text { Syphilis prevalence rate } \\
\text { (OPD) (d) }\end{array}$ & $\begin{array}{l}\text { Percentage of all STI } \\
\text { patients tested who tested } \\
\text { positive for syphilis using a }\end{array}$ & Impact & $\checkmark$ & & & & & & & \\
\hline
\end{tabular}


Table 2 Unique indicators identified ( $n=132)$ (Continued)

\begin{tabular}{|c|c|c|c|c|c|c|c|c|c|c|c|}
\hline \multirow[t]{2}{*}{ Domain } & \multirow[t]{2}{*}{$\begin{array}{l}\text { Indicator (Sets it Appears } \\
\text { In) } \\
\text { [italics = appears in } \\
\text { multiple sets] }\end{array}$} & \multirow[t]{2}{*}{ Definition (Sets) } & \multirow[t]{2}{*}{ Type } & \multicolumn{5}{|c|}{ Data Source } & \multicolumn{3}{|c|}{$\begin{array}{l}\text { Overlap with } \\
\text { Priority } \\
\text { Indicators }\end{array}$} \\
\hline & & & & Facilities & $\begin{array}{l}\text { Service } \\
\text { Providers }\end{array}$ & $\begin{array}{l}\text { Affected } \\
\text { Population }\end{array}$ & $\begin{array}{l}\text { Program } \\
\text { Records }\end{array}$ & $\begin{array}{l}\text { Not } \\
\text { Specified }\end{array}$ & SDG & GS & $\begin{array}{l}\text { Core } \\
\text { WHO }\end{array}$ \\
\hline & & pre-qualified syphilis test & & & & & & & & & \\
\hline STI & $\begin{array}{l}\text { Help-seeking behaviors } \\
\text { for treating selected STI- } \\
\text { associated symptoms (e) }\end{array}$ & $\begin{array}{l}\text { Percentage of women who } \\
\text { had unusual genital } \\
\text { discharge and/or genital } \\
\text { ulcers or sores in the last } \\
12 \text { months who went to a } \\
\text { health facility for treatment }\end{array}$ & Outcome & & & $\checkmark$ & & & & & \\
\hline STI & $\begin{array}{l}\text { Knowledge of selected } \\
\text { STI-associated symptoms } \\
\text { (e) }\end{array}$ & $\begin{array}{l}\text { Percentage of women of } \\
\text { reproductive age who } \\
\text { know at least one of three } \\
\text { selected STI-associated } \\
\text { symptoms }\end{array}$ & Output & & & $\checkmark$ & & & & & \\
\hline STI & Partner tracing $(\mathrm{d})$ & $\begin{array}{l}\text { Percentage of positive } \\
\text { syphilis cases where } \\
\text { contacts were tested }\end{array}$ & Output & $\checkmark$ & & & & & & & \\
\hline STI & $\begin{array}{l}\text { Access to STI services } \\
\text { since disaster (c) }\end{array}$ & Not Specified & Output & & & $\checkmark$ & & & & & \\
\hline STI & $\begin{array}{l}\text { Primary healthcare } \\
\text { facilities with } \\
\text { antimicrobials to provide } \\
\text { syndromic management } \\
\text { to patients presenting } \\
\text { with symptoms of an STI } \\
\text { (f) }\end{array}$ & Not Specified & Output & $\checkmark$ & & & & & & & \\
\hline STI & $\begin{array}{l}\text { STI/RTI case } \\
\text { management (b) }\end{array}$ & $\begin{array}{l}\text { Percentage of total number } \\
\text { of patients with STI/RTI } \\
\text { accessing services that are } \\
\text { assessed, treated and } \\
\text { counselled according to } \\
\text { protocol }\end{array}$ & Outcome & $\checkmark$ & & & & & & & \\
\hline STI & $\begin{array}{l}\text { STI/RTI management } \\
\text { skills of service providers } \\
\text { (b) }\end{array}$ & $\begin{array}{l}\text { Percentage of service } \\
\text { providers trained (or } \\
\text { retrained) to manage STI/ } \\
\text { RTI cases according to } \\
\text { protocol }\end{array}$ & Process & & $\checkmark$ & & & & & & \\
\hline
\end{tabular}

${ }^{1}$ Priority indicator sets include: The Sustainable Development Goal Monitoring Framework Indicators (SDG); the Global Strategy for Women, Children and Adolescents Monitoring and Evaluation Framework Indicators (GS); and the WHO's Global Reference List of 100 Core Health Indicators (Core) a: MISP Indicators

b: Comprehensive Reproductive Health Service Indicators

c: Indicators for Pregnant and Postpartum Women after Disaster

d: Health Information System Standards and Indicators

e: Reproductive Health Assessment Toolkit for Conflict-Affected Women Key Indicator List

f: Sphere Standards Indicators

g: Response Monitoring Indicator List for Maternal, Newborn and Child Health and Nutrition in Emergencies

h: OCHA Indicators Registry

had definitions which were consistent with the definition of the priority indicator.

\section{Discussion}

Findings from this review provided a comprehensive look at the existing indicators recommended for use for the purpose of M\&E of SRH in humanitarian settings. Results clearly showed substantial variations across the different indicator sets in terms of the SRH domains covered, highlighted the different approaches taken towards data collection, and demonstrated discrepancies in indicator definitions across sets. The lack of consistency of coverage and definitions across indicator sets clearly indicates the need for greater harmonization. Differences in coverage observed across indicator domains may in part be due to changing emphases within the field of SRH over time. For example, both $\mathrm{ARH}$ and $\mathrm{CAC}$ are relatively newer areas of focus within SRH and are more politically challenging in comparison to other domains; this means there has been less time for and more challenges involved in developing indicators for these 


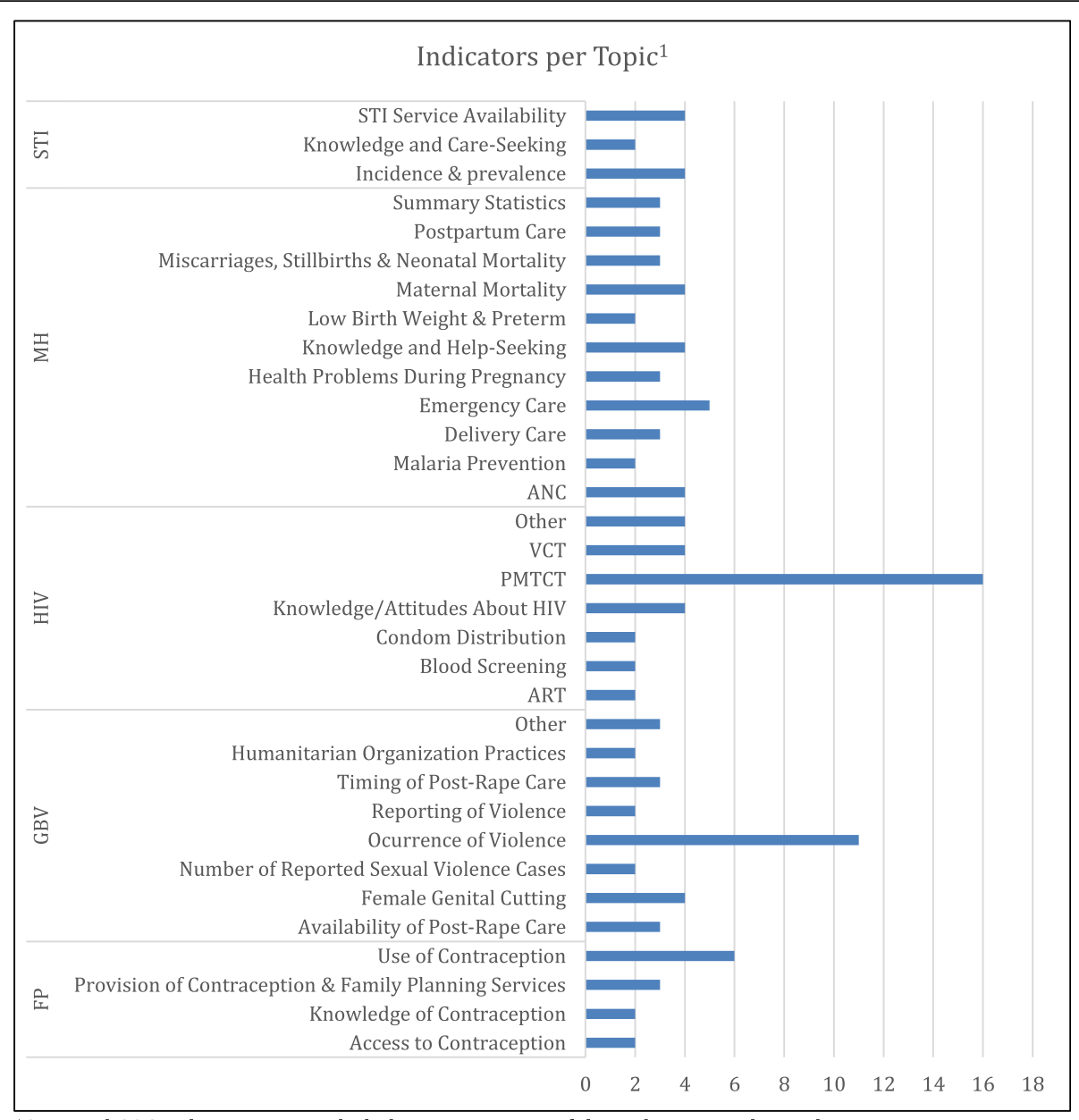

${ }^{1} \mathrm{ARH}$ and CAC indicators not included since coverage of these domains is limited

Fig. 2 Coverage of topics within each SRMH domain across all unique indicators

domains [15]. Regardless, these discrepant findings in the numbers and types of M\&E indicators across the different SRH domains suggest the need for critically appraising the extent to which these domains should be covered during routine monitoring and evaluation, and whether development of additional indicators may be needed for adequate coverage for SRH in humanitarian settings.

Notable in their absence from the literature were indicator sets from many of the organizations that commonly implement relief efforts in emergency settings. Despite searching the websites of 85 organizations (many of which implement relief efforts), only one indicator set published by an implementing agency was identified [26]. This indicates that many organizations that provide SRH services in humanitarian settings do not make their M\&E frameworks or indicator sets available in the public domain. Consequently, it is difficult to know which indicators are actually regularly used and reported on [4].
Broadly, our findings concur with the conclusions of Checchi et al. [4] in their review of public health information methods for crisis-affected populations. They assert the need for a common set of crisis-specific public health indicators, as well as establishment of a single health information platform for use in emergencies and a global data repository to store and analyze the data collected [4]. These needs underlie the consultative review process led by the WHO's Department of Reproductive Health and Research (HRP) which aims to establish a recommended core set of SRH indicators for humanitarian settings.

Findings from this literature review have fed directly into the WHO's consultative review process. The identified indicators, especially the 28 that overlapped with one or more of the priority indicator sets (i.e. either the SDG, GS or Core WHO indicators) (Table 4), served as a starting basis for the review process during a Technical Consultation with experts and stakeholder convened in December 2018. A report describing the progress of the consultative process, 


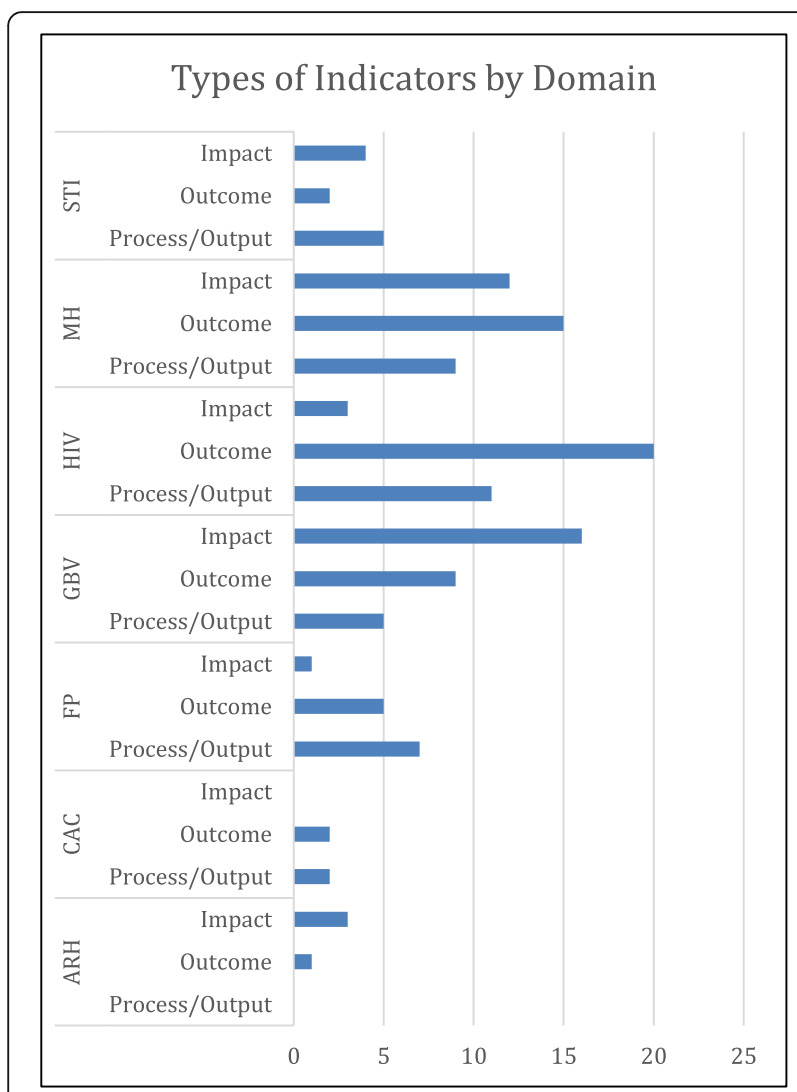

Fig. 3 Indicator types by SRMH domain across all unique indicators

including results regarding indicator prioritization and standardization, were circulated to all partners who participated in the Technical Consultation and will be made available on the HRP website (https://www.who.int/ reproductivehealth/publications/) upon incorporation of input from partners.

The prioritization process during the Technical Consultation revolved around selecting those indicators identified in this review which appeared in multiple sets and also overlapped with SDG, and/or GS, and/or WHO 100 Core Indicators. Along with prioritization, the review process focused on standardization-resolving inconsistencies across indicators sets and establishing clearly defined numerators, denominators, and data collection guidance for each indicator based on input from SRH experts and other stakeholders. Additionally, given the lack of indicator sets from implementing agencies identified in this review (as noted above), representatives from key implementing agencies participated in the Technical Consultation and contributed information about their internal M\&E indicators and processes.

Ongoing areas of focus during the WHO's consultative review process are indicator coverage and feasibility of usage. As this literature review demonstrates, coverage of existing indicators across domains varies substantially, and differs by indicator set. This raises questions regarding what the coverage of a core set of indicators should be, and what is most realistic. For example, this literature review identified few indicators in domains such as ARH and CAC-domains often associated with pertinent socio-political challenges that might prevent or hamper data collection. Another major feasibility question is not only whether the data collection for obtaining certain indicators would be logistically possible, but also whether it would be politically and bureaucratically feasible, making harmonization of indicators across settings difficult. Results from this literature review also indicate an uneven balance of indicators by data source, with the vast majority drawing on data from facilities or population-based surveys. Yet indicators drawing on other data sources, such as community-based indicators, may be more appropriate and informative for assessing services provided at levels beyond the health facility. Finally, for some SRH domains, such as HIV and GBV, crucial services are often provided by separate programs specific to these domains which are distinct from SRH services and programs. Therefore, ensuring appropriate coverage of the HIV and GBV domains within a core set of indicators will require multi-sector collaboration on the indicator selection process. These and other issues related to establishing a core SRH indicator set for humanitarian settings will continue to be explored during stakeholder consultations and via field-testing to assess indicator feasibility via collection of real-time data across varying humanitarian contexts.

In addition to the indicators identified, this review's descriptions of the data collection tools, processes, and guidance that currently exist in association with each indicator set could be useful for identifying data collection platforms to scale up and harmonize data collection and reporting of indicators across agencies, settings, and time, as called for by Checchi et al. [4]. The extent to which supporting resources are available for data collection, analysis and reporting currently varies substantially across indicator sets. For instance, the indicator set identified to have the most extensive set of supporting resources is the UNHCR's Health Information System Standards and Indicators, which is part of the Twine system (accessible at http://twine.unhcr.org/ app/). The Twine system not only includes data collection tools and data entry templates, but also provides a mechanism for centralized reporting and automatic analysis. This is also the only set of indicators that is associated with an established system for ongoing data collection.

There is a need for greater emphasis on monitoring and evaluating SRH in humanitarian settings comprehensively, rather than taking a siloed approach. Only a few M\&E studies examining a multi-domain set of 
Table 3 Indicators Appearing in Multiple Sets $(n=33)$

\begin{tabular}{|c|c|c|c|}
\hline Domain & Indicator & Number of Sets it Appears $\ln ^{1}$ & Definitions Same Across Sets ${ }^{1}$ \\
\hline ARH & Proportion of STIs among those under 18 years & 2 & Yes \\
\hline FP & Contraceptive prevalence & 3 & No \\
\hline GBV & Number of reported rape cases & 2 & No \\
\hline GBV & Number of reported sexual violence cases & 2 & Yes \\
\hline GBV & Timing of EC provision & 3 & No \\
\hline GBV & Timing of PEP provision & 3 & No \\
\hline GBV & Timing of STI prophylaxis & 2 & No \\
\hline GBV & Availability of clinical management of rape survivors & 4 & No \\
\hline HIV & Quality of blood donation screening & 3 & No \\
\hline HIV & Condom use & 2 & No \\
\hline HIV & Timing of PEP & 2 & Yes \\
\hline HIV & PMTCT ARV coverage (mother-infant pair) & 2 & Yes \\
\hline HIV & Condom distribution rate & 2 & No \\
\hline HIV & PMTCT pre-test counselling coverage & 2 & Yes \\
\hline HIV & PMTCT post-test counselling and result & 2 & Yes \\
\hline HIV & Coverage of supplies for standard precautions & 2 & Yes \\
\hline HIV & VCT post-test counselling and result & 2 & Yes \\
\hline $\mathrm{MH}$ & Proportion of low birth weight & 3 & No \\
\hline $\mathrm{MH}$ & Neonatal mortality rate & 2 & Yes \\
\hline $\mathrm{MH}$ & Stillbirth rate & 2 & Yes \\
\hline $\mathrm{MH}$ & Complete ANC coverage & 3 & No \\
\hline $\mathrm{MH}$ & Coverage of syphilis screening & 2 & Yes \\
\hline $\mathrm{MH}$ & Tetanus vaccination coverage & 2 & Yes \\
\hline $\mathrm{MH}$ & Percentage of caesarean section & 4 & Yes \\
\hline $\mathrm{MH}$ & Delivery assisted by a skilled attendant & 4 & No \\
\hline $\mathrm{MH}$ & EmOC services utilization & 2 & Yes \\
\hline $\mathrm{MH}$ & Postnatal care coverage & 3 & No \\
\hline $\mathrm{MH}$ & Coverage of clean delivery kits & 3 & No \\
\hline $\mathrm{MH}$ & BEmOC services availability & 2 & Yes \\
\hline $\mathrm{MH}$ & CEmOC services availability & 2 & Yes \\
\hline $\mathrm{MH}$ & Investigation of maternal deaths & 2 & Yes \\
\hline STI & Incidence of genital ulcer disease & 2 & Yes \\
\hline STI & Incidence of male urethral discharge & 2 & Yes \\
\hline
\end{tabular}

$\mathrm{SRH}$ indicators were found in the peer-reviewed or grey-literature [14, 17, 22-25]. Instead, many studies focused on one single domain, such as $M H$ or GBV, which hinders a general understanding of the status of SRH services and outcomes in humanitarian settings as a whole. The exception were those studies which examined the MISP implementation [14, 17, 23-25]. More specifically, the MISP Process Evaluation toolkit could be considered a valuable tool, given its broad coverage of multiple SRH domains across the six main MISP objectives. It should be noted, however, that although this toolkit is valuable, the data generated is focused on assessment of implementation processes, rather than M\&E of SRH services and outcomes over time, or across settings.

Several strengths can be attributed to this review. These include its rigorous adherence to the PRISMA guidelines and the in-depth mapping process undertaken to synthesize key information from the 179 indicators identified. Additionally, focusing on the indicators themselves as the unit of analysis allowed for a unique and illuminating analysis. There are several limitations that should be equally noted. First, most of the indicator sets identified were either from guidance 
Table 4 Indicators Overlapping with Those in Priority Indicator Sets $(n=28)$

\begin{tabular}{|c|c|c|c|}
\hline Domain & Indicator & $\begin{array}{l}\text { Priority Indicator Sets with } \\
\text { Overlap }^{\mathrm{a}}\end{array}$ & $\begin{array}{l}\text { Definition Same as in Priority } \\
\text { Indicator Set }\end{array}$ \\
\hline ARH & Proportion of births among those under 18 years & SDG, GS, Core & No \\
\hline FP & Contraceptive prevalence & Core & No \\
\hline FP & Modern contraceptive prevalence & Core & No \\
\hline GBV & IPV in past year & SDG, GS, Core & Yes \\
\hline GBV & Prevalence of female genital cutting & SDG, GS & No \\
\hline GBV & Timing of PEP provision & GS & Yes \\
\hline GBV & Availability of clinical management of rape survivors & GS & Yes \\
\hline HIV & Number on ART & GS, Core & No \\
\hline HIV & Condom use & Core & No \\
\hline HIV & PMTCT ARV coverage (mother) & Core & No \\
\hline HIV & PMTCT ARV coverage (mother-infant pair) & Core & No \\
\hline HIV & $\begin{array}{l}\text { Receipt of ARV drugs for PMTCT by pregnant women known to be } \\
\text { HIV positive }\end{array}$ & Core & Yes \\
\hline $\mathrm{MH}$ & Proportion of low birth weight & Core & Yes \\
\hline $\mathrm{MH}$ & Maternal mortality ratio & SDG, GS, Core & Yes \\
\hline $\mathrm{MH}$ & Neonatal mortality rate & SDG, GS, Core & Yes \\
\hline $\mathrm{MH}$ & Stillbirth rate & GS, Core & Yes \\
\hline $\mathrm{MH}$ & Complete ANC coverage & GS, Core & No \\
\hline $\mathrm{MH}$ & Coverage of syphilis screening & GS & Yes \\
\hline $\mathrm{MH}$ & Coverage of intermittent presumptive treatment for malaria & Core & No \\
\hline $\mathrm{MH}$ & Delivery assisted by a skilled attendant & SDG, GS, Core & Yes \\
\hline $\mathrm{MH}$ & Postnatal care coverage & GS, Core & No \\
\hline $\mathrm{MH}$ & BEmOC services availability & GS & No \\
\hline $\mathrm{MH}$ & CEmOC services availability & GS & No \\
\hline $\mathrm{MH}$ & EmOC services availability & GS & Yes \\
\hline $\mathrm{MH}$ & Investigation of maternal deaths & Core & No \\
\hline STI & Incidence of genital ulcer disease & Core & No \\
\hline STI & Incidence of male urethral discharge & Core & No \\
\hline STI & Selected STI-associated symptoms in the past 12 months & Core & No \\
\hline
\end{tabular}

apriority indicator sets include: The Sustainable Development Goal Monitoring Framework Indicators (SDG); the Global Strategy for Women, Children and Adolescents Monitoring and Evaluation Framework Indicators (GS); and the WHO's Global Reference List of 100 Core Health Indicators (Core)

${ }^{b}$ When an indicator appeared in multiple sets and had multiple definitions, this question was marked yes if any of those definitions was the same as that in the priority indicator set

bodies (i.e. the Sphere Project, or the IAWG) or peerreview published literature, rather than directly reported from implementing agencies. As discussed above, this makes it difficult to accurately reflect the realities of M\&E data collection efforts by the different implementing agencies from the field. Additionally, this review also does not indicate the feasibility and the practicality of collecting particular indicators in particular settings. Instead, feasibility will be assessed via field-testing at later stage in the WHO's consultative review process. Finally, we only included English language studies. However, considering the global nature of this topic, we expect only very few eligible studies are missed by excluding non-English literature.
In addition to the consultative process currently underway, further research is needed to address these gaps, such as supplementing this information with field experience on what is being collected at the field level as well as seeking global consensus and a process of prioritization of a core list of M\&E SRH indicators in humanitarian settings. Future studies should systematically examine the extent to which indicators are measuring what should be measured, vs. what can be measured, and which indicators and data collection methods are appropriate for use in which settings. Additionally, iterative participatory consultative processes, engaging a wide variety of stakeholders involved in humanitarian response-particularly those most connected to on-the-ground realities coupled with feasibility 
assessments-will be an essential component to culminate these efforts to standardize and harmonize indicators and to ensure scale up, accountability and commitment of partners to collecting some or all of the recommended M\&E indicators.

\section{Conclusions}

The results of this review assert the need for standardizing data collection efforts for M\&E of SRH services and outcomes in humanitarian settings. A core list of indicators is essential for assessing response status over time as well as across and within countries. The 28 indicators identified via this review which overlap with either the SDGs, the Global Strategy or the 100 WHO Core indicators have provided the starting basis for an extensive consultative review process which aims to establish a standardized core indicator list. Rigorous reporting on a core list of indicators is a prerequisite for making the investment case that SRH response in humanitarian settings saves lives. Efforts are underway to conceptualize a core set of SRH indicators as well as to test their measurement feasibility. A standardized definition of accountability is a crucial bi-product of these efforts. A commitment by agencies on a core set of indicators requires a more conscious effort as well as willingness to share information and coordinate efforts. This could be possible by scaling up M\&E of SRH efforts within the WHO's global health cluster, as it could ensure measurement sustainability, especially so for protracted crises.

\section{Additional file}

Additional file 1: Additional Supporting Resources and Guiding MESH terms. (DOCX $49 \mathrm{~kb}$ )

\section{Abbreviations \\ ARH: Adolescent reproductive health; CAC: Comprehensive abortion care; FP: Family planning; GBV: Gender-based violence; GS: GLOBAL Strategy for Women's Children's and Adolescents' Health; HIV: Human immunodeficiency virus; IAWG: Inter-Agency Working Group; M\&E: Monitoring and evaluation; $\mathrm{MH}$ : Maternal and newborn health; MISP: Minimum initial service package; PRISMA-P: Preferred reporting items for systematic review and meta-analysis protocols; SDGs: Sustainable Development Goals; SRH: Sexual and reproductive health; STI: Sexually transmitted infection; WHO: World Health Organization}

\section{Acknowledgements}

Not applicable.

\section{Authors' contributions}

ETB-S developed the study protocol, carried out the literature search, led the screening and data extraction processes, and was a major contributor in writing the manuscript. LK conceptualized the study, oversaw study protocol development and the literature search process, and was a major contributor in writing the manuscript. OU assisted with screening and data extraction and contributed to writing the manuscript. LS contributed to study conceptualization, oversaw study protocol development, and contributed to writing the manuscript. All authors read and approved the final manuscript.

\section{Funding}

This work was funded by the Department of Reproductive Health and Research (RHR), including the UNDP/UNFPA/UNICEF/WHO/World/Bank Special programme of research, development and research training in human reproduction (HRP). The funders had no role in study design, data collection and analysis, decision to publish, or preparation of the manuscript.

Availability of data and materials

Not applicable, however study protocol is registered with PROSPERO (CRD42018108492).

Ethics approval and consent to participate

Not applicable.

\section{Consent for publication}

Not applicable.

\section{Competing interests}

The authors declare that they have no competing interests. The authors alone are responsible for the views expressed in this [article] and they do not necessarily represent the views, decisions or policies of the institutions with which they are affiliated.

\section{Author details}

${ }^{1}$ Horizons Global Health Consulting, Eagle, CO, USA. ${ }^{2}$ Department of Reproductive Health and Research, World Health Organization, Geneva, Switzerland. ${ }^{3}$ Independent Consultant, New Delhi, India.

Received: 22 November 2018 Accepted: 31 July 2019

Published online: 30 September 2019

\section{References}

1. Pillai V, Wang YC, Maleku A. Women, war, and reproductive health in developing countries. Soc Work Health Care. 2017;56(1):28-44.

2. Inter-agency Working Group on Reproductive Health in Crises. Inter-agency field manual on reproductive health in humanitarian settings: 2010 revision for field review: Inter-agency Working Group on Reproductive Health in Crises; 2010.

3. Sphere Project. Sphere handbook: humanitarian charter and minimum standards in disaster response: the sphere Project; 2011.

4. Checchi F, Warsame A, Treacy-Wong V, Polonsky J, van Ommeren M, Prudhon C. public health information in crisis-affected populations: a review of methods and their use for advocacy and action. Lancet (London, England). 2017;390(10109):2297-313.

5. Onyango MA, Hixson BL, MCNally S. Minimum initial service package (MISP) for reproductive health during emergencies: time for a new paradigm? Glob Public Health. 2013:8(3):342-56.

6. Moher D, Shamseer L, Clarke M, Ghersi D, Liberati A, Petticrew M, et al. Preferred reporting items for systematic review and meta-analysis protocols (PRISMA-P) 2015 statement. Systematic reviews. 2015;4(1):1.

7. Leadership Council of the Sustainable Development Solutions Network. Indicators and a monitoring framework for the sustainable development goals: Launchng a data revolution for the SDGs. Sustainable Development Solutions Network, vol. 2015.

8. Every Woman Every Child. Indicator and Monitoring Framework for the Global Strategy for Women's Children's and Adolescents' Health (2016-2030). Every Woman Every Child; 2016.

9. World Health Organization. Global reference list of 100 Core health indicators. Geneva: World Health Organization; 2015.

10. CARE International. Care emergency toolkit: sexual and reproductive health: CARE international; 2017 [Available from: https://www.careemergencytoolkit. org/core-sectors/26-sexual-and-reproductive-health/.

11. Centers for Disease Control and Prevention (CDC). Reproductive health assessment toolkit for conflict-affected women. Atlanta: Division of Reproductive Health, National Center for Chronic Disease Prevention and Health Promotion, Coordinating Center for Health Promotion, Centers for Disease Control and Prevention, Department of Health and Human Services; 2007.

12. Centers for Disease Control and Prevention (CDC). A process evaluation of the reproductive health assessment (RHA) toolkit for conflict-affected women: a report of findings, recommendations, and next steps. Atlanta: Division of Reproductive Health, National Center for Chronic Disease Prevention and Health Promotion, Coordinating Center for Health 
Promotion, Centers for Disease Control and Prevention, Department of Health and Human Services; 2011.

13. Centers for Disease Control and Prevention (CDC). Health indicators for disaster-affected pregnant and postpartum women and infants. Atlanta: Division of Reproductive Health, National Center for Chronic Disease Prevention and Health Promotion, Coordinating Center for Health Promotion, Centers for Disease Control and Prevention, Department of Health and Human Services; 2013.

14. Doedens W, Giga N, Krause S, Onyango MA, Sami S, Stone E, et al. Reproductive health Services for Syrian Refugees in Zaatri refugee camp and Irbid City, Jordan: an evaluation of the minimum initial service package: Boston University School of public health, United Nations Population Fund, US Centers for Disease Control and Prevention, Women's Refugee Commission; 2013

15. Inter-agency Working Group (IAWG) on Reproductive Health in Crises. Interagency field manual on reproductive health in humanitarian settings. Geneva: Inter-agency Working Group on Reproductive Health in Crises; 2010.

16. Inter-agency Working Group (IAWG) on Reproductive Health in Crises. MISP Process Evaluation Tools 2017 Available from: http://iawg.net/resource/ misp-process-evaluation-tools-2017/.

17. Krause S, Williams H, Onyango MA, Sami S, Doedens W, Giga N, Stone E, Tomczyk B. Reproductive health services for Syrian refugees in Zaatri Camp and Irbid City, Hashemite Kingdom of Jordan: an evaluation of the Minimum Initial Services Package. Conflict Health. 2015;9(Suppl 1 Taking Stock of Reproductive Health in Humanitarian):S4.

18. Moreland S, Curran J. A Guide for Monitoring and Evaluating PopulationHealth-Environment Programs, Second Edition. Chapel Hill: MEASURE Evaluation, University of North Carolina; 2018.

19. Pyone T, Dickinson F, Kerr R, Boschi-Pinto C, Mathai M, van den Broek N. Data collection tools for maternal and child health in humanitarian emergencies: a systematic review. Bull World Health Organ. 2015;93(9):648-58a-m.

20. UN OCHA. Indicator Registry 2016. Available from: https://ir.hpc.tools/. Accessed May 2019.

21. UNHCR. Health information system (HIS) standards and indicators guide 2010. Available from: http://www.unhcr.org/protection/health/4614 ab8e2/53-standards-indicators-guide-revised.html. Accessed May 2019.

22. Whitmill J, Blanton C, Doraiswamy S, Cornier N, Schilperood M, Spiegel P, Tomczyk B. Retrospective analysis of reproductive health indicators in the United Nations high commissioner for refugees post-emergency camps 2007-2013. Confl Health. 2016;10:13.

23. Women's Commission for Refugee Women and Children. Reproductive health coordination gap, services ad hoc: minimum initial service package (MISP) assessment in Kenya: The Women's Commission for Refugee Women and Children, International Rescue Committee; 2008.

24. Women's Refugee Commission. Evaluation of the MISP for reproductive health Services in Post-earthquake Nepal: Women's Refugee Commission; 2016.

25. Women's Refugee Commission, CARE International. International Planned Parenthood Federation, save the children. Priority reproductive health activities in Haiti: an inter-agency MISP assessment: Women's Refugee Commission; 2011.

26. World Vision International. Guide to maternal, newborn and Child health and nutrition in emergencies: World Vision International; 2012.

27. Zotti ME, Williams AM. Reproductive health assessment after disaster: introduction to the RHAD toolkit. J Women's Health. 2011;20(8):1123-7.

28. Zotti ME, Williams AM, Wako E. Post-disaster health indicators for pregnant and postpartum women and infants. Matern Child Health J. 2015;19(6):1179-88.

\section{Publisher's Note}

Springer Nature remains neutral with regard to jurisdictional claims in published maps and institutional affiliations.

Ready to submit your research? Choose BMC and benefit from:

- fast, convenient online submission

- thorough peer review by experienced researchers in your field

- rapid publication on acceptance

- support for research data, including large and complex data types

- gold Open Access which fosters wider collaboration and increased citations

- maximum visibility for your research: over $100 \mathrm{M}$ website views per year

At BMC, research is always in progress.

Learn more biomedcentral.com/submissions 